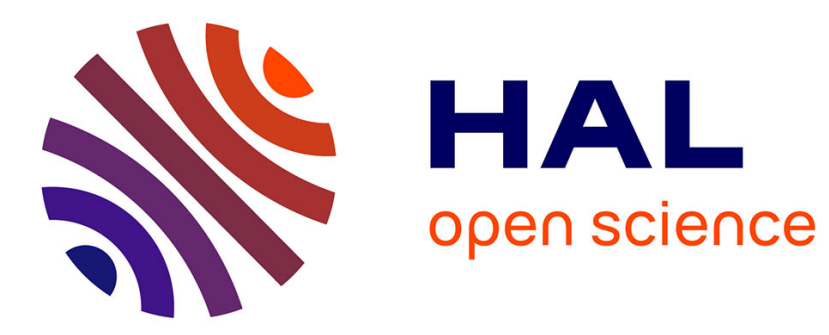

\title{
Mathematical analysis of a chemotaxis-type model of soil carbon dynamic.
}

\author{
Alaaeddine Hammoudi, Oana Iosifescu
}

\section{To cite this version:}

Alaaeddine Hammoudi, Oana Iosifescu. Mathematical analysis of a chemotaxis-type model of soil carbon dynamic.. Chinese Annals of Mathematics - Series B, 2018, 39 (2), pp.253-280. 10.1007/s11401018-1063-7 . hal-01818727

\section{HAL Id: hal-01818727 \\ https://hal.science/hal-01818727}

Submitted on 19 Jun 2018

HAL is a multi-disciplinary open access archive for the deposit and dissemination of scientific research documents, whether they are published or not. The documents may come from teaching and research institutions in France or abroad, or from public or private research centers.
L'archive ouverte pluridisciplinaire HAL, est destinée au dépôt et à la diffusion de documents scientifiques de niveau recherche, publiés ou non, émanant des établissements d'enseignement et de recherche français ou étrangers, des laboratoires publics ou privés. 


\title{
Mathematical analysis of a chemotaxis-type model of soil carbon dynamic.
}

\author{
Alaaeddine Hammoudi *, Oana Iosifescu *
}

\begin{abstract}
The goal of this paper is to study the mathematical properties of a new model of soil carbon dynamics which is a reaction-diffusion system with a chemotactic term, with the aim to account for the formation of soil aggregations in the bacterial and microorganism spatial organization (hot spot in soil). This is a spatial and chemotactic version of MOMOS (Modelling Organic changes by Micro-Organisms of Soil), a model recently introduced by M. Pansu and his group. We present here two forms of chemotactic terms, first a "classical" one and second a function which prevents the overcrowding of microorganisms. We prove in each case the existence of a nonnegative global solution, we investigate its uniqueness and the existence of a global attractor for all the solutions.
\end{abstract}

Keywords: Soil organic carbon dynamics, reaction-diffusion-advection system, positive weak solutions, periodic weak solutions.

Mathematics Subject Classification 2000: 35B09, 35B10, 35D30, 35K51, 35Q92.

\section{Introduction.}

Chemotaxis is the ability of some bacteria to direct their movement according to the gradient of chemicals contained in their environment. In soil, some bacteria microorganisms that degrade organic carbon (SOC) are motile and chemotactic. This phenomenon is observed in experiments [1] and on field. Nevertheless to our best knowledge no model of terrestrial carbon cycle adresses this issue. Indeed, these models are essentially compartimental

*Institut de mathématiques - IMAG, Université Montpellier, Case courier 051, Place Eugène Bataillon, 34095 Montpellier Cedex 5, France, alaaeddine.hammoudi@univmontp2.fr, iosifescu@math.univ-montp2.fr 
corresponding naturally to systems of ordinary differential equations (e.g Century, RothC, MOMOS) [2]. They are used globally to estimate soil $\mathrm{CO}_{2}$ emissions in local land management and crop optimization, among other things.

Very few prototypes of spatial soil organic model have been proposed. Some of them use systems of partial differential equations: Balesdent et al. [3] combined vertical directed transport of organic carbon with a degradation phenomenon and diffusion. More recently, Goudjo et al. [4] proposed a three dimensional model for dissolved organic matter using also a system of PDEs. Other authors opted for a finite sequence of interconnected systems of ordinary differential equations each localized in a soil layer (see [5]).

We previously studied the model MOMOS proposed by Pansu (see [6],[7]), which is a nonlinear system of ordinary differential equations [8] written as:

$$
\dot{\mathbf{y}}=\mathbf{g}(t, \mathbf{y})
$$

where

$$
\mathbf{y}=\left(\begin{array}{c}
u \\
v \\
w
\end{array}\right)
$$

and

$$
\mathbf{G}(t, \mathbf{y})=\left(\begin{array}{c}
-k_{1}(t) u-q(t) u^{2}+k_{2}(t) v+k_{3}(t) w+f(t) \\
k_{1}(t) u-\left(k_{2}(t)+k_{4}(t)\right) v \\
k_{4}(t) v-k_{3}(t) w
\end{array}\right)
$$

In these equations the unknown $u$ models the alive microbial biomass, whereas the unknowns $v$ and $w$ are soil organic matters with distinct decomposition rates.

In reality, the nonnegative functions $k_{i} i \in\{1,2,3,4\}, q$ and $f$ depend not only on time but also on space because of the variability in soil clay content. The phenomena described by MOMOS can also be subjected to the influence of transport and sedimentation through transport and diffusion. In order to test the effect of soil heterogeneity we studied in [9] the following reaction-diffusion-advection initial problem:

$$
\left\{\begin{array}{l}
\frac{\partial u_{i}}{\partial t}-\operatorname{div}\left(\mathbf{A}_{i}(t, x) \nabla u_{i}\right)+\mathbf{B}_{i}(t, x) \nabla u_{i}=g_{i}^{+}(t, x, \mathbf{u}),(t, x) \in Q_{T}:=(0, T) \times \Omega \\
\gamma\left(\mathbf{A}_{i}(t, x) \nabla u_{i}\right) \cdot \nu+\beta_{i}(t, x) u_{i}=0, \quad(t, x) \in \Sigma_{T}:=(0, T) \times \partial \Omega \\
u_{i}(0)=u_{i, 0} \quad \text { in } \Omega
\end{array}\right.
$$

where $\Omega$ is a domain in $\mathbb{R}^{n}$ representing the soil, $\mathbf{A}_{i}$ is a diffusion matrix and $\mathbf{B}_{i}$ a transport vector, for each $i=1,2,3$. 
In [9] the boundary conditions were either of Dirichlet type $\left(\gamma=0, \beta_{i} \equiv 1\right)$ or of Neumann-Robin type $\left(\gamma=1, \beta_{i}(t, x) \geq 0\right)$. The right hand side term of (1.1) was :

$$
\mathbf{g}^{+}(t, x, \mathbf{u}):=\left(\begin{array}{c}
-k_{1}(t, x) u_{1}-q(t, x)\left|u_{1}\right| u_{1}+k_{2}(t, x) u_{2}+k_{3}(t, x) u_{3}+f(t, x) \\
k_{1}(t, x) u_{1}-\left(k_{2}(t, x)+k_{4}(t, x)\right) u_{2} \\
k_{4}(t, x) u_{2}-k_{3}(t, x) u_{3}
\end{array}\right)
$$

where we replaced the term $q(t, x) u_{1}^{2}$ with $q(t, x)\left|u_{1}\right| u_{1}$ for more accuracy, since $q(t, x) u_{1}$ corresponds to a kinetic coefficient that cannot be negative. We assumed there that the diffusion matrices $\mathbf{A}_{i}$ were bounded, symmetric and coercive:

$$
\mathbf{A}_{i} \in L^{\infty}\left(Q_{T}\right)^{n \times n},
$$

$$
\mathbf{A}_{i}(t, x) \zeta \cdot \zeta \geq c|\zeta|^{2}, \quad \forall \zeta \in \mathbb{R}^{n} \text {, a.e in } Q_{T}, \text { with } c>0
$$

and the transport vectors $\mathbf{B}_{i}$ were bounded on $Q_{T}$ :

$$
\mathbf{B}_{i} \in L^{\infty}\left(Q_{T}\right)^{N}, \quad\left|\left(\mathbf{B}_{i}(t, x)\right)_{j}\right| \leq c_{\max } \text { a.e in } Q_{T}, \text { for all } 1 \leq j \leq n .
$$

Also we assumed that the functions $k_{j}, \beta_{i}$ and $q$ were nonnegative and bounded, i.e. for all $j=1,2,3,4$ and $i=1,2,3$

$$
\begin{gathered}
k_{j}, q \in L^{\infty}\left(Q_{T}\right), \quad 0 \leq k_{j}(t, x), q(t, x) \leq C_{\max } \text { a.e on } Q_{T}, \\
\beta_{i} \in L^{\infty}\left(\Sigma_{T}\right), \quad 0 \leq \beta_{i}(t, x) \leq C_{\max } \text { a.e on } \Sigma_{T},
\end{gathered}
$$

where constant $C_{\max }>0$. Finally we assumed that the initial data and input were nonnegative and bounded :

$$
u_{i, 0} \in L_{+}^{2}(\Omega), \quad f \in L_{+}^{2}\left(Q_{T}\right), \quad f(t, x) \leq C_{\max } \text { a.e } \text { on } Q_{T} .
$$

In [9] we proved first that this model had a unique weak solution. We were looking for weak solutions, because initial inputs were not regular enough to give rise to more "regular" solutions. Second, for periodic data, we proved the existence of a maximal and a minimal periodic solution of this system. In some particular cases, the minimal and the maximal periodic solutions coincide and this function becomes a global attractor for any bounded solution of the periodic system.

In the present work a new PDEs model is considered to take account of chemotaxis. The chemotactic movement of bacteria to root exudates is well known to play an important role in rhizosphere colonisation. Field studies 
with tracers and laboratory experiments using soil columns were both used to demonstrate the effect of chemotaxis on microbial movements. So, the model proposed here can represent the spatial heterogeneity of soil microbial biomass, highlighted by recent observations at submicron scale [1]

The new model derived from a simplified MOMOS ODEs model, which comprised only two differential equations instead of the three originally, where the microbial biomass was $u$ and the organic matter was $v$. As additional simplifying hypothesis soil temperature, soil moisture, soil texture and organic input were considered to be isotropic and constant with time. Hence, the simplified ODEs model can be expressed as:

$$
\left\{\begin{array}{l}
\dot{u}=-k_{1} u-q u^{2}+k_{2} v \\
\dot{v}=-k_{2} v+k_{1} u+f
\end{array}\right.
$$

with the initial conditions $\left(u_{0}, v_{0}\right)$, where $k_{1}$ is the microbial mortality rate, $k_{2}$ is the soil carbon degradation rate, $q$ is the metabolic quotient and $f$ is the soil organic carbon input. It can be proven that the unique positive steady state $\left(u_{0}^{*}, v_{0}^{*}\right)$, is stable [8].

The chemotaxis-type model was finded following the conventional KellerSegel approach [10], using an advection-diffusion system. This comprised two parabolic equations in a smooth domain with no-flux boundary conditions. The advection term was controlled by the gradient of the chemoattractant. Applying the same principles to our problem leads to the following reaction-diffusion-chemotaxis system $\left(P_{h}\right)$

$$
\left\{\begin{array}{l}
\partial_{t} u-a \Delta u=-\beta \operatorname{div}(h(u) \nabla v)-k_{1} u-q|u| u+k_{2} v, \quad(t, x) \in Q_{T} \\
\partial_{t} v-d \Delta v=-k_{2} v+k_{1} u+f, \quad(t, x) \in \Omega_{T}, \\
\nabla u \cdot \nu=\nabla v \cdot \nu=0, \quad(t, x) \in \Sigma_{T}, \\
u(0)=u_{0}, \quad \text { in } \Omega, \\
v(0)=v_{0}, \quad \text { in } \Omega .
\end{array}\right.
$$

The parameter $\beta$ is the chemotaxis sensitivity, $a$ and $d$ are the diffusion coefficients of microbes and soil organic carbon respectively, $\Omega$ is a smooth and bounded domain, and $h(\cdot)$ is a continuous function, involved in the modelling of chemotaxis. As bacteria can release exoenzymes to avoid overcrowding, the function $h$ can be selected to limit overcrowding, as required. This new model is, therefore, a new variation of the Keller-Segel approach [10] with the reaction part modified to fit the MOMOS model. In the first equation of $\left(P_{h}\right)$, we change again the term $q u^{2}$ by $q|u| u$, see [9]. 
We prove here (see Appendix 1) the existence of Turing patterns that may provide possible explanations for the formation of soil aggregations, for the bacterial and micro-organisms spatial organizations (hotspots in soil) or justify the formation of the microscopic patterns observed by Vogel et al. [1]. Although spatial heterogeneity can be verified visually in a numerical simulation (see Appendix 2), formal mathematical analysis is required to confirm its emergence and to provide a mathematical proof of the necessary conditions. The mathematical criteria are based on matrices derived from equations and analysed using conditions on the determinant, trace and eigenvalues.

Keller-Segel model was the earliest mathematical system involving chemotaxis [10]. Many others models emerged specially in biology and ecology. Most authors focused their efforts essentially on existence and on asymptotic behaviour of solutions in one or two dimensional domains in order to avoid blow-up of solutions (see [11], [12], [13], [14], [15] and references therein).

Unlike the classical Keller-Segel model, where equations are coupled only by the chemotactic term, the system of partial differential equations $\left(P_{h}\right)$ is also coupled through the reaction term. More specifically, the organic matter will not only attract microorganisms, but part of it will be "transformed", under a degradation process, to microorganisms. This mechanism introduces a supplementary linear coupling term in the first equation of this model. Many authors ([11], [12], [13] and references therein) already considered reaction coupling terms, but under some restrictive conditions, which are not verified here. This feed-back in the chemotactic equation is not compatible with mass conservation of microorganisms, unlike in [12], [15]. Furthermore, neither the boundedness of the microorganisms total mass nor the positivity and the boundedness (existence of threshold) of the solution remain immediate, unlike in [13], [11] and [14].

Our main concern here is to prove the existence of a unique solution to this minimal MOMOS model improved by adding chemotaxis effect. We consider two chemotactic functions $h$, a "classical" one, $h(u)=u$, and a second one which prevents overcrowding of microorganisms, $h(u)=u(M-u)$ if $0 \leq u \leq M$ and zero otherwise, proposed by Wrzosek [15].

This paper is organized as follows. Section 2 introduces some notations, results and tools used throughout the paper. Section 3 presents sufficient conditions to get global solutions, and to prove the existence of an exponential attractor, in the case where $h(u)=u$. Section 4 is concerned with the 
second chemotactic function, where the chemotactic term cancels when $u$ achieves the threshold $M$, which helps to prove that any local solution is actually global. In sections 3 and 4 the domain is two dimensional. In Section 5 , still keeping the second form of $h$ and for domains of dimension less than or equal to 3 (the dimension 3 is particularly interesting in applications), we prove the existence of a unique solution, with less restrictions on the initial conditions and forcing term than in section 4. In Appendix 1we prove that chemotactic term in system $\left(P_{h}\right)$ is mandatory to obtain Turing patterns and in Appendix 2 we give some numerical simulations.

\section{Mathematical preliminary and notations.}

Unless it is explicitly indicated, $\Omega$ is a bounded region in $\mathbb{R}^{2}$ of $\mathcal{C}^{3}$ class, the constants $a, \beta, q, d, k_{1}$ and $k_{2}$ are nonnegative, and $f$ is a nonegative function belonging to an admissible space to be fixed later. In all that follows $C$ denotes a positive constant which may vary from line to line.

We recall here some known results (see [16], [17] and references therein) that will help afterwards.

Interpolation space:

For $0 \leq s_{0}<s<s_{1}<\infty, H^{s}(\Omega)$ is the interpolation space $\left[H^{s_{0}}(\Omega), H^{s_{1}}(\Omega)\right]_{\theta}$ with $s=(1-\theta) s_{0}+\theta s_{1}$ between $H^{s_{0}}(\Omega)$ and $H^{s_{1}}(\Omega)$. Furthermore, we have

$$
\|\cdot\|_{H^{s}} \leq\|\cdot\|_{H^{s_{0}}}^{1-\theta}\|\cdot\|_{H^{s_{1}}}^{\theta} .
$$

Embedding theorem:

When $0<s<1, H^{s}(\Omega) \subset L^{p}(\Omega)$ for $\frac{1}{p}=\frac{1-s}{2}$ with the estimate

$$
\|\cdot\|_{L^{p}} \leq C_{s}\|\cdot\|_{H^{s}}
$$

When $s=1, H^{1}(\Omega) \subset L^{q}(\Omega)$ for any $1 \leq q<\infty$ and

$$
\|\cdot\|_{L^{q}} \leq C_{q, p}\|\cdot\|_{H^{1}}^{1-p / q}\|\cdot\|_{L^{p}}^{p / q}
$$

where $1 \leq p \leq q<\infty$.

When $s>1 H^{s}(\Omega) \subset C(\bar{\Omega})$ with continuous embedding.

Fractional Power of the Laplace operator: (see ([17], Chap 2.7), [12])

Let $a_{0}, a_{1}>0$ be constants and $L=-a_{1} \Delta+a_{0}$ be the Laplace operator equipped with the Newman boundary conditions, with the domain $\mathcal{D}(L)=$ 
$\left\{u \in H^{2}(\Omega) ; \frac{\partial u}{\partial \nu}=0\right.$ on $\left.\partial \Omega\right\}=H_{N}^{2}(\Omega)$. Thus $L$ is a positive definite selfadjoint operator of $L^{2}(\Omega)$. For $\theta>0$, the fractional power on $L$ is defined and noted $L^{\theta}$ and $L^{\theta}$ is also a positive definite self-adjoint operator on $L^{2}(\Omega)$. More

$$
\mathcal{D}\left(L^{\theta}\right)= \begin{cases}H^{2 \theta}(\Omega), & 0 \leq \theta<\frac{3}{4}, \\ H_{N}^{2 \theta}(\Omega), & \frac{3}{4}<\theta \leq \frac{3}{2},\end{cases}
$$

with the norm equivalence.

\section{Some useful inequalities}

Biler's lemma (see [18])

Let $0 \leq u \in H^{1}(\Omega)$ and $N_{l o g}^{1}(u):=\|(u+1) \log (u+1)\|_{L^{1}}$. For any $\eta>0$,

$$
\|u\|_{L^{3}}^{3} \leq \eta\|u\|_{H^{1}} N_{l o g}^{1}(u)+p\left(\eta^{-1}\right)\|u\|_{L^{1}},
$$

where $p(\cdot)$ denotes here some increasing function.

Let $\varepsilon \in(0,1]$. It is proved in $([13],(2.10) \div(2.12))$ that:

$$
\begin{array}{cl}
\|\nabla(u \nabla v)\|_{L^{2}} \leq C_{\varepsilon}\|u\|_{H^{1}}\|v\|_{H^{2+\varepsilon}}, & \text { for all } u \in H^{1}(\Omega), v \in H^{2+\varepsilon}(\Omega) \\
\|\nabla(u \nabla v)\|_{L^{2}} \leq C_{\varepsilon}\|u\|_{H^{1+\varepsilon}}\|v\|_{H^{2}}, & \text { for all } u \in H^{1+\varepsilon}(\Omega), v \in H^{2}(\Omega) \\
\|\nabla(u \nabla v)\|_{H^{1}} \leq C\|u\|_{H^{2}}\|v\|_{H^{3}}, & \text { for all } u \in H^{2}(\Omega), v \in H^{3}(\Omega) .
\end{array}
$$

Local Existence

We need first to prove the existence of local solution of $\left(P_{h}\right)$. For this purpose, we use the result obtained by Yagi and based on the Galerkin method (see [12], [17]).

Let $V$ and $H$ be seperable Hilbert spaces with dense and compact embedding $V \subset H$. Let $V^{\prime}$ be the dual space of $V$ and identify $H$ and $H^{\prime}$ to get:

$$
V \subset H \subset V^{\prime}
$$

The duality product between $V$ and $V^{\prime}$ is denoted by $\left.<\cdot, \cdot\right\rangle$. It coincides with the scalar product on $H$ denoted by $(\cdot, \cdot)$.

Consider the following Cauchy problem of a semilinear abstract differential equation:

$$
\begin{aligned}
& \frac{d U}{d t}+A U=G(U)+F(t), 0<t \leq T \\
& U(0)=U_{0}
\end{aligned}
$$


in the space $V^{\prime}$.

Here, $A$ is the positive definite self-adjoint operator of $H$ defined by a symmetric sesquilinear form $a(U, \tilde{U})$ on $V$, with $<A U, \tilde{U}>_{V, V^{\prime}}=a(U, \tilde{U})$.

Assumptions on $a(\cdot, \cdot)$ :

$$
\begin{aligned}
& \text { (a.i) } \quad\|a(U, \tilde{U})\|_{H} \leq M\|U\|_{V}\|\tilde{U}\|_{V}, \quad U, \tilde{U} \in V, \\
& \text { (a.ii) } \quad a(U, U) \geq \delta\|U\|_{V}^{2}, \quad U \in V,
\end{aligned}
$$

with constants $\delta, M>0$. The operator $A$ is also bounded from $V$ to $V^{\prime}$.

Assumptions on $G(\cdot)$ :

$G($.$) is a continuous function from V$ to $V^{\prime}$, which satisfy:

(g.i) For each $\zeta>0$, there exists an increasing continuous function $\phi_{\zeta}:[0, \infty) \rightarrow[0, \infty)$ such that:

$$
\|G(U)\|_{V^{\prime}} \leq \zeta\|U\|_{V}+\phi_{\zeta}\left(\|U\|_{H}\right), \quad U \in V
$$

(g.ii) For each $\zeta>0$, there exists an increasing continuous function $\psi_{\zeta}$ : $[0, \infty) \rightarrow[0, \infty)$ such that:

$$
\begin{aligned}
& \|G(U)-G(\tilde{U})\|_{V^{\prime}} \leq \zeta\|U-\tilde{U}\|_{V} \\
& \quad+\psi_{\zeta}\left(\|U\|_{H}+\|\tilde{U}\|_{H}\right) \times\|U-\tilde{U}\|_{H}\left(\|U\|_{V}+\|\tilde{U}\|_{V}+1\right), \quad U, \tilde{U} \in V
\end{aligned}
$$

Finally $F(\cdot) \in L^{2}\left(0, T ; V^{\prime}\right)$ is a given function and $U_{0} \in H$ is an initial value. Then, we have [12]:

Theorem 2.1. Under assumptions (a.i), (a.ii), (g.i) and (g.ii) and for every $F(\cdot) \in L^{2}\left(0, T ; V^{\prime}\right)$ and $U_{0} \in H$, there exists a unique local solution $U$ of (2.7) such that:

$$
U \in H^{1}\left(0, T\left(U_{0}, F\right) ; V^{\prime}\right) \cap C\left(\left[0, T\left(U_{0}, F\right)\right] ; H\right) \cap L^{2}\left(0, T\left(U_{0}, F\right), V\right)
$$

Here $T\left(U_{0}, F\right)$ is determined by the norm $\left\|U_{0}\right\|_{H}$ and $\|F\|_{L^{2}\left(0, T ; V^{\prime}\right)}$

\section{First case: $h(u)=u$.}

\subsection{Local existence and positivity.}

Let $\varepsilon_{0}$ arbitrarily fixed, $\varepsilon_{0} \in(0,1)$. Then: 
Theorem 3.1. Let $u_{0} \in L^{2}(\Omega), v_{0} \in H^{1+\varepsilon_{0}}(\Omega)$ and $f \in L^{2}\left(0, T ; H^{\varepsilon_{0}}(\Omega)\right)$ be nonnegative functions. Then $\left(P_{h}\right)$ has a unique nonnegative local solution on an interval $\left[0, T_{0}\right]$ such that

$$
\begin{aligned}
& u \in H^{1}\left(0, T_{0} ; H^{1}(\Omega)^{\prime}\right) \cap C\left(\left[0, T_{0}\right] ; L^{2}(\Omega)\right) \cap L^{2}\left(0, T_{0}, H^{1}(\Omega)\right), \\
& v \in H^{1}\left(0, T_{0} ; H^{\varepsilon_{0}}(\Omega)\right) \cap C\left(\left[0, T_{0}\right] ; H^{1+\varepsilon_{0}}(\Omega)\right) \cap L^{2}\left(0, T_{0}, H_{N}^{2+\varepsilon_{0}}(\Omega)\right),
\end{aligned}
$$

where $T_{0}$ depends only on $\|f\|_{L^{2}\left(0, T ; H^{\left.\varepsilon_{0}(\Omega)\right)}\right.}$, $\left\|u_{0}\right\|_{L^{2}(\Omega)}$ and $\left\|v_{0}\right\|_{H^{1+\varepsilon_{0}(\Omega)}}$.

Proof. First Step: Construction of a unique local solution

Let $A_{1}=-a \Delta+k_{1}$ and $A_{2}=-d \Delta+k_{2}$ be two operators with the same domain $H_{N}^{2}(\Omega)$. $A_{1}$ and $A_{2}$ are positive self-adjoint operators on $L^{2}(\Omega)$. We can then define their corresponding fractional power operators (see [17], as described in the previous section.

Let $V=H^{1}(\Omega) \times H_{N}^{2+\varepsilon_{0}}(\Omega)$ and $H=L^{2}(\Omega) \times H^{1+\varepsilon_{0}}(\Omega)$. Identifying $H$ with its dual space gives: $V \subset H=H^{\prime} \subset V^{\prime}$ and $V^{\prime}=\left(H^{1}(\Omega)\right)^{\prime} \times H^{\varepsilon_{0}}(\Omega)$ with the duality product:

$$
\left.<U, \tilde{U}>_{V, V^{\prime}}=<u, \tilde{u}>_{H^{1},\left(H^{1}\right)^{\prime}}+<A_{2}^{1+\frac{\varepsilon_{0}}{2}} v, A_{2}^{\frac{\varepsilon_{0}}{2}} \tilde{v}\right)>_{L^{2}, L^{2}},
$$

where $U=(u, v) \in V$ and $\tilde{U}=(\tilde{u}, \tilde{v}) \in V^{\prime}$.

We also set a symmetric sesquilinear form on $V \times V$,

$$
a(U, \tilde{U})=\int_{\Omega}\left\{a \nabla u \cdot \nabla \tilde{u}+k_{1} u \tilde{u}\right\} d x+\left(A_{2}^{1+\frac{\varepsilon_{0}}{2}} v, A_{2}^{1+\frac{\varepsilon_{0}}{2}} \tilde{v}\right)_{L^{2}}
$$

for $U=(u, v)$ and $\tilde{U}=(\tilde{u}, \tilde{v}) \in V$.

This forms is in fact a linear isomorphism $A$ from $V$ to $V^{\prime}$ :

$$
A=\left(\begin{array}{cc}
A_{1} & 0 \\
0 & A_{2}
\end{array}\right)
$$

and $A$ becomes a positive definite self-adjoint operator in $H$.

Finally let $f(\cdot) \in L^{2}\left(0, T, H^{\varepsilon_{0}}(\Omega)\right)$ and let $G: V \rightarrow V^{\prime}$ be the mapping:

$$
G(U):=\left(\begin{array}{c}
\beta \nabla(u \nabla v)-q|u| u+k_{2} v \\
k_{1} u
\end{array}\right),
$$

with $U=(u, v) \in V$.

Then $\left(P_{h}\right)$ is the following semilinear differential equation:

$$
\begin{aligned}
& \frac{d U}{d t}+A U=G(U)+F(t) \quad \text { in } V^{\prime}, \quad 0<t \leq T, \\
& U(0)=U_{0},
\end{aligned}
$$


where $F(t)=\left(\begin{array}{c}0 \\ f(t)\end{array}\right)$.

In order to apply the existence result of Theorem 2.1. to problem (3.1), let us verify the assumptions on $a(\cdot, \cdot)$ and $G(\cdot)$.

The assumptions on $a(\cdot, \cdot)$ are classically satisfied (see for example [12]).

For the conditions on $G$ we have that for an arbitrary $U=(u, v) \in V$ and $\delta>0$ :

$$
\begin{aligned}
\|\nabla \cdot(u \nabla v)\|_{\left(H^{1}\right)^{\prime}} & \leq C\|u\|_{L^{4}}\|\nabla v\|_{L^{4}} \leq\|u\|_{L^{2}}^{\frac{1}{2}}\|u\|_{H^{1}}^{\frac{1}{2}}\|v\|_{H^{1}}^{\frac{1}{2}}\|v\|_{H^{2}}^{\frac{1}{2}} \\
& \leq\|u\|_{L^{2}}^{\frac{1}{2}}\|u\|_{H^{1}}^{\frac{1}{2}}\|v\|_{H^{1+\varepsilon_{0}}}^{\frac{1+\varepsilon_{0}}{2}}\|v\|_{H^{2+\varepsilon_{0}}}^{\frac{1-\varepsilon_{0}}{2}} \\
& \leq C\|U\|_{H}^{1+\frac{\varepsilon_{0}}{2}}\|U\|_{V}^{1-\frac{\varepsilon_{0}}{2}} \leq \zeta\|U\|_{V}+\phi_{\zeta}\left(\|U\|_{H}\right)
\end{aligned}
$$

and

$$
\|v\|_{\left(H^{1}\right)^{\prime}} \leq C\|U\|_{H}, \quad\left\|u^{2}\right\|_{\left(H^{1}\right)^{\prime}} \leq C\|u\|_{L^{3}}^{2} \leq \zeta\|u\|_{H^{1}}+\phi_{\zeta}\left(\|u\|_{L^{2}}\right) .
$$

Finally it is clear that:

$$
\|u\|_{H^{\varepsilon_{0}}} \leq \zeta\|u\|_{H^{1}}+C_{\zeta}\left(\|u\|_{L^{2}}\right) .
$$

All these inequalities show that the condition (g.i) is fullfiled.

From the embedding theorem we have:

$$
\begin{aligned}
& \left|\int_{\Omega}(\tilde{u}-u) \nabla v \cdot \nabla \rho d x\right| \leq C\|\tilde{u}-u\|_{L^{2}}\|\tilde{v}\|_{H^{2+\varepsilon_{0}}}\|\rho\|_{H^{1}} \\
& \left|\int_{\Omega} \nabla(\tilde{v}-v) u \cdot \nabla \rho d x\right| \leq C\|u\|_{H^{1}}\|\tilde{v}-v\|_{H^{1+\varepsilon_{0}}}\|\rho\|_{H^{1}}
\end{aligned}
$$

and using the interpolation theorem and Young inequality we obtain:

$$
\begin{aligned}
\|u-\tilde{u}\|_{H^{\varepsilon_{0}}} & \leq C\|U-\tilde{U}\|_{V}^{\varepsilon_{0}}\|U-\tilde{U}\|_{H}^{1-\varepsilon_{0}} \\
& \leq \zeta\|U-\tilde{U}\|_{V}+C_{\zeta}\|U-\tilde{U}\|_{H}
\end{aligned}
$$

for an arbitrary $\zeta>0$. On the other hand we have that:

$$
\begin{aligned}
\|u|u|-\tilde{u}|\tilde{u}|\|_{\left(H^{1}\right)^{\prime}} & \leq C\left(\|(|u|-|\tilde{u}|) u\|_{L^{3 / 2}}+\|(u-\tilde{u})|\tilde{u}|\|_{L^{3 / 2}}\right) \\
& \leq C\|u-\tilde{u}\|_{L^{2}}\left(\|u\|_{L^{6}}+\|\tilde{u}\|_{L^{6}}\right)
\end{aligned}
$$

All these inequalities permit to show that condition (g.ii) is fullfiled too.

Second Step: Positivity of the solution

Now let us take the following semilinear system:

$$
\begin{gathered}
\frac{d U}{d t}+A U=\tilde{G}(U)+F(t), 0<t \leq T \\
U(0)=U_{0},
\end{gathered}
$$


where $A, F$ and $Y_{0}$ are defined as previously, and the mapping $\tilde{G}: V \rightarrow V^{\prime}$ is defined by:

$$
\tilde{G}(U):=\left(\begin{array}{c}
\beta \nabla(u \nabla v)-q|u| u+k_{2}|v| \\
k_{1} u
\end{array}\right) .
$$

By Theorem 2.1. there exists a local solution $U=(u, v)$ on $\left[0, T_{0}\right] \times \Omega$, with $T_{0}$ depending only on $U_{0}$ and $F$. Let us define $u^{+}=\max (u, 0)$ and $u^{-}=\max (-u, 0)$.

We multiply the first equation by $-u^{-}$and we integrate in space. So

$$
\begin{aligned}
\frac{1}{2} \frac{d}{d t}\left\|u^{-}\right\|_{L^{2}}^{2}+a\left\|\nabla u^{-}\right\|_{L^{2}}^{2}+k_{1}\left\|u^{-}\right\|_{L^{2}}^{2} & \leq \int_{\Omega} \beta u^{-} \nabla v \nabla u^{-} d x \\
& \leq \beta\|\nabla v\|_{L^{\infty}} \int_{\Omega} u^{-}\left|\nabla u^{-}\right| d x
\end{aligned}
$$

for $0<t \leq T_{0}$.

Using Young inequality we get

$$
\frac{1}{2} \frac{d}{d t}\left\|u^{-}\right\|_{L^{2}}^{2}+a\left\|\nabla u^{-}\right\|_{L^{2}}^{2} \leq\|\nabla v\|_{L^{\infty}}\left(C_{\varepsilon}\left\|u^{-}\right\|_{L^{2}}^{2}+\varepsilon\left\|\nabla u^{-}\right\|_{L^{2}}^{2}\right)
$$

with $\varepsilon>0$ small enough and $C_{\varepsilon}>0$.

Taking $\varepsilon=\frac{a}{\|\nabla v\|_{L^{\infty}}}$ we get

$$
\begin{aligned}
\frac{d}{d t}\left\|u^{-}\right\|_{L^{2}}^{2} & \leq C\|\nabla v\|_{L^{\infty}}^{2}\left\|u^{-}\right\|_{L^{2}}^{2} \\
& \leq C\|v\|_{H^{2+\varepsilon_{0}}}^{2}\left\|u^{-}\right\|_{L^{2}}^{2}
\end{aligned}
$$

Since $v \in L^{2}\left(0, T_{0} ; H^{2+\varepsilon_{0}}(\Omega)\right)$ and $\left\|u_{0}^{-}\right\|_{L^{2}}^{2}=0$ by Gronwall Lemma we deduce that $u$ is nonnegative on $\left[0, T_{0}\right]$. By classical results on linear parabolic equations $v$ is nonnegative on $\left[0, T_{0}\right]$ too. So, the nonnegative solution $U$ of (3.2) is also a solution of (3.1).

Remark. If initial conditions $U_{0}$ and data $f$ are not positive, this theorem proves anyway the existence of a local solution. However, as this is an ecology model, only nonnegative solutions make sense.

With minor changes due to our different problem $\left(P_{h}\right)$,we prove as in [13] the following theorems. 
Theorem 3.2. Let $U_{0}=\left(u_{0}, v_{0}\right) \in H^{1}(\Omega) \times H_{N}^{2}(\Omega)$ and $f \in L^{2}\left(0, T ; H^{1}(\Omega)\right)$. Then there exists a unique local solution $U=(u, v)$ of $(3.1)$ on an interval $\left[0, T_{U_{0}, f}\right]$ such that

$$
\begin{aligned}
& u \in H^{1}\left(0, T_{U_{0}, f} ; L^{2}(\Omega)\right) \cap C\left(\left[0, T_{U_{0}, f}\right] ; H^{1}(\Omega)\right) \cap L^{2}\left(0, T_{U_{0}, f}, H_{N}^{2}(\Omega)\right), \\
& v \in H^{1}\left(0, T_{U_{0}, f} ; H^{1}(\Omega)\right) \cap C\left(\left[0, T_{U_{0}, f}\right] ; H_{N}^{2}(\Omega)\right) \cap L^{2}\left(0, T_{U_{0}, f}, H_{N}^{3}(\Omega)\right),
\end{aligned}
$$

where $T_{U_{0}, f}$ is determined by $\|f\|_{L^{2}\left(0, T ; H^{1}(\Omega)\right)}$, $\left\|u_{0}\right\|_{H^{1}(\Omega)}$ and $\left\|v_{0}\right\|_{H^{2}(\Omega)}$.

Theorem 3.3. Let $U_{0}=\left(u_{0}, v_{0}\right) \in H_{N}^{2}(\Omega) \times H_{N}^{3}(\Omega)$ and $f \in L^{2}\left(0, T ; H_{N}^{2}(\Omega)\right)$. Then there exists a unique local solution $U=(u, v)$ to $(3.1)$ on an interval $\left[0, T_{U_{0}, f}\right]$ such that:

$$
\begin{aligned}
& u \in H^{1}\left(0, T_{U_{0}, f} ; H^{1}(\Omega)\right) \cap C\left(\left[0, T_{U_{0}, f}\right] ; H_{N}^{2}(\Omega)\right) \cap L^{2}\left(0, T_{U_{0}, f}, H_{N}^{3}(\Omega)\right), \\
& v \in H^{1}\left(0, T_{U_{0}, f} ; H_{N}^{2}(\Omega)\right) \cap C\left(\left[0, T_{U_{0}, f}\right] ; H_{N}^{3}(\Omega)\right) \cap L^{2}\left(0, T_{U_{0}, f}, D\left(A_{2}^{2}\right)(\Omega)\right),
\end{aligned}
$$

where $T_{U_{0}, f}$ is determined by $\|f\|_{L^{2}\left(0, T ; H^{2}(\Omega)\right)}$, $\left\|u_{0}\right\|_{H^{2}(\Omega)}$ and $\left\|v_{0}\right\|_{H^{3}(\Omega)}$.

\subsection{Global existence.}

This section is devoted to proving the following result:

Theorem 3.4. Let $\varepsilon_{0} \in(0,1)$ and let $u_{0} \in L^{2}(\Omega), v_{0} \in H^{1+\varepsilon_{0}}(\Omega)$ and $f \in L^{2}\left(0, T, H^{1}(\Omega)\right) \cap L^{\infty}\left(0, T ; L^{2}(\Omega)\right)$ be nonnegative functions. Then there exists a unique global and nonnegative solution $(u, v)$ for the system $\left(P_{h}\right)$ with $h(u)=u$ such that:

$$
\begin{aligned}
& u \in H^{1}\left(0, T ;\left(H^{1}(\Omega)\right)^{\prime}\right) \cap C\left([0, T] ; L^{2}(\Omega)\right) \cap L^{2}\left(0, T ; H^{1}(\Omega)\right) \\
& v \in H^{1}\left(0, T ; H^{\varepsilon_{0}}(\Omega)\right) \cap C\left([0, T] ; H^{1+\varepsilon_{0}}(\Omega)\right) \cap L^{2}\left(0, T ; H_{N}^{2+\varepsilon_{0}}(\Omega)\right) .
\end{aligned}
$$

Proof. We proceed in two steps.

First Step

We show that $\|v\|_{H^{1}(\Omega)}$ and $N_{l o g}^{1}(u)=\|(u+1) \log (u+1)\|_{L^{1}(\Omega)}$ are bounded for all $t \in\left[0, T_{0}\right]$.

We consider the function $\log (u+1)$; since $\nabla \log (u+1)=\frac{\nabla u}{u+1}$, it follows that $\log (u+1) \in L^{2}\left(0, T_{0} ; H^{1}(\Omega)\right)$. Noting that

$$
\frac{d}{d t} \int_{\Omega}\{(u+1) \log (u+1)-u\} d x=<\frac{d u}{d t}, \log (u+1)>_{H^{1},\left(H^{1}\right)^{\prime}}
$$


we obtain from the first equation of $\left(P_{h}\right)$ multiplied by $\log (u+1)$ that:

$$
\begin{aligned}
\frac{d}{d t} \int_{\Omega}\{(u+1) \log (u+1)-u\} d x+4 a \int_{\Omega}|\nabla \sqrt{u+1}|^{2} d x \\
=\beta \int_{\Omega} \frac{u}{u+1} \nabla u \nabla v d x+\int_{\Omega}\left(-k_{1} u-q u^{2}+k_{2} v\right) \log (u+1) d x .
\end{aligned}
$$

So using Stokes theorem we deduce:

$$
\int_{\Omega} \frac{u}{u+1} \nabla u \nabla v d x=\int_{\Omega}(\log (u+1)-u) \Delta v d x \leq \frac{\eta}{2}\|\Delta v\|_{L^{2}(\Omega)}^{2}+\frac{1}{2 \eta}\|u\|_{L^{2}(\Omega)}^{2} .
$$

Since

$$
\left(k_{1} u+q u^{2}\right) \log (u+1) \geq k_{1}((u+1) \log (u+1)-u),
$$

if we denote $\Psi(t)=\|(u+1) \log (u+1)-u\|_{L^{1}(\Omega)}$, we get

$$
\frac{d}{d t} \Psi(t)+k_{1} \Psi(t) \leq \frac{\eta}{2}\|\Delta v\|_{L^{2}(\Omega)}^{2}+\left(\frac{k_{2}^{2}}{2 \varepsilon}+\frac{\beta^{2}}{2 \eta}\right)\|u\|_{L^{2}(\Omega)}^{2}+\frac{\varepsilon}{2}\|v\|_{L^{2}(\Omega)}^{2},
$$

with arbitary $\varepsilon, \eta>0$.

From the second equation of $\left(P_{h}\right)$ multiplied respectively by $v$ and $\Delta v$ we obtain that

$$
\begin{aligned}
& \frac{1}{2} \frac{d}{d t} \int_{\Omega} v^{2}+d \int_{\Omega}|\nabla v|^{2} d x+k_{2} \int_{\Omega} v^{2} d x=k_{1} \int_{\Omega} u v d x+\int_{\Omega} v f d x \\
\leq & \left(\frac{k_{1} A}{2}+\frac{B}{2}\right)\|v\|_{L^{2}(\Omega)}^{2}+\frac{k_{1}}{2 A}\|u\|_{L^{2}(\Omega)}^{2}+\frac{1}{2 B}\|f\|_{L^{2}(\Omega)}^{2}, \text { with arbitrary } A, B>0,
\end{aligned}
$$

and

$$
\begin{aligned}
& \frac{1}{2} \frac{d}{d t} \int_{\Omega}|\nabla v|^{2}+d \int_{\Omega}|\Delta v|^{2} d x+k_{2} \int_{\Omega}|\nabla v|^{2} d x=k_{1} \int_{\Omega} u \Delta v d x+\int_{\Omega} f \Delta v d x \\
\leq & \left(\frac{k_{1} C}{2}+\frac{D}{2}\right)\|\Delta v\|_{L^{2}(\Omega)}^{2}+\frac{k_{1}}{2 C}\|u\|_{L^{2}(\Omega)}^{2}+\frac{1}{2 D}\|f\|_{L^{2}(\Omega)}^{2}, \text { with arbitrary } C, D>0 .
\end{aligned}
$$

Choosing $\varepsilon=d, \eta=k_{2}, A=\frac{k_{2}}{2 k_{1}}, B=\frac{k_{2}}{2}, C=\frac{d}{2 k_{1}}, D=\frac{d}{2}$, we deduce

$$
\begin{aligned}
\frac{d}{d t}(\Psi(t)+ & \left.\|v\|_{H^{1}(\Omega)}^{2}\right)+\frac{d}{2}\|\Delta v\|_{L^{2}(\Omega)}^{2}+k_{2}\|v\|_{H^{1}(\Omega)}^{2}+k_{1} \Psi(t) \\
& \leq\left(\frac{k_{1}^{2}}{k_{2}}+\frac{\beta^{2}}{2 k_{2}}+\frac{k_{2}^{2}}{2 d}+\frac{k_{1}^{2}}{d}\right)\|u\|_{L^{2}(\Omega)}^{2}+\left(\frac{1}{k_{2}}+\frac{1}{d}\right)\|f\|_{L^{2}(\Omega)}^{2}
\end{aligned}
$$


By addition of the first two equation of $\left(P_{h}\right)$ it follows that:

$$
\frac{d}{d t}\left(\|u\|_{L^{1}(\Omega)}+\|v\|_{L^{1}(\Omega)}\right)+q\|u\|_{L^{2}(\Omega)}^{2}=\|f\|_{L^{1}(\Omega)}
$$

which implies that for all $t \in\left[0, T_{0}\right]$ we have the inequality :

$$
\begin{aligned}
& \|u(t)\|_{L^{1}(\Omega)}+\|v(t)\|_{L^{1}(\Omega)} \\
& \quad \leq\left\|u_{0}\right\|_{L^{1}(\Omega)}+\left\|v_{0}\right\|_{L^{1}(\Omega)}+\int_{0}^{t}\|f(s)\|_{L^{1}(\Omega)} d s
\end{aligned}
$$

As $N_{\log }^{1}(u)=\int_{\Omega}(u+1) \log (u+1) d x$ we have $\Psi(t)=N_{\log }^{1}(u(t))-\|u(t)\|_{L^{1}(\Omega)}$. Let denote $\delta:=\max \left(1, \frac{1}{q}\left(\frac{k_{1}^{2}}{k_{2}}+\frac{\beta^{2}}{2 k_{2}}+\frac{k_{2}^{2}}{2 d}+\frac{k_{1}^{2}}{d}\right)\right)$ and $\sigma:=\min \left(k_{1}, k_{2}\right)>0$. Therefore from (3.3), (3.4) and (3.5) we obtain the following inequality:

$$
\begin{aligned}
& \frac{d}{d t}\left(N_{\text {log }}^{1}(u(t))+\|v(t)\|_{H^{1}(\Omega)}^{2}+(\delta-1)\|u(t)\|_{L^{1}(\Omega)}+\delta\|v(t)\|_{L^{1}(\Omega)}\right) \\
& \quad+\sigma\left(N_{l o g}^{1}(u(t))+\|v(t)\|_{H^{1}(\Omega)}^{2}+(\delta-1)\|u(t)\|_{L^{1}(\Omega)}+\delta\|v(t)\|_{L^{1}(\Omega)}\right) \\
& \leq \sigma(\delta+1)\left(\left\|u_{0}\right\|_{L^{1}(\Omega)}+\left\|v_{0}\right\|_{L^{1}(\Omega)}\right) \\
& \quad+\delta\|f(t)\|_{L^{1}(\Omega)}+\left(\frac{1}{k_{2}}+\frac{1}{d}\right)\|f(t)\|_{L^{2}(\Omega)}^{2}+\sigma \delta \int_{0}^{t}\|f(s)\|_{L^{1}(\Omega)} d s
\end{aligned}
$$

We denote by $g(t)=N_{\text {log }}^{1}(u(t))+\|v(t)\|_{H^{1}(\Omega)}^{2}+(\delta-1)\|u(t)\|_{L^{1}(\Omega)}+\delta\|v(t)\|_{L^{1}(\Omega)}$.

Since $g(t)$ satisfies the following ordinary differential inequality:

$$
\begin{aligned}
& g^{\prime}(t)+\sigma g(t) \\
& \leq \\
& \quad \sigma(\delta+1)\left(\left\|u_{0}\right\|_{L^{1}(\Omega)}+\left\|v_{0}\right\|_{L^{1}(\Omega)}\right) \\
& \quad+\delta\|f(t)\|_{L^{1}(\Omega)}+\left(\frac{1}{k_{2}}+\frac{1}{d}\right)\|f(t)\|_{L^{2}(\Omega)}^{2}+\sigma \delta \int_{0}^{t}\|f(s)\|_{L^{1}(\Omega)} d s=C,
\end{aligned}
$$

with $C>0$ depending only on $\|f\|_{L^{\infty}\left(0, T ; L^{2}(\Omega)\right)},\left\|u_{0}\right\|_{L^{2}(\Omega)}$ and $\left\|v_{0}\right\|_{H^{1+\varepsilon_{0}(\Omega)}}$, we get

$$
g(t) \leq e^{-\sigma t} g(0)+C, \text { for all } t \geq 0 .
$$

Thus the inequality

$$
\begin{aligned}
& N_{\log }^{1}(u(t))+\|v(t)\|_{H^{1}(\Omega)}^{2} \\
& \quad \leq N_{\log }^{1}\left(u_{0}\right)+\left\|v_{0}\right\|_{H^{1}(\Omega)}^{2}+(\delta-1)\left\|u_{0}\right\|_{L^{1}(\Omega)}+\delta\left\|v_{0}\right\|_{L^{1}(\Omega)}+C,
\end{aligned}
$$


holds for all $t \in\left[0, T_{0}\right]$, where the last constant $C>0$ is independent of $T_{0}$ and depends only on $\|f\|_{L^{\infty}\left(0, T ; L^{2}(\Omega)\right)},\left\|u_{0}\right\|_{L^{2}(\Omega)}$ and $\left\|v_{0}\right\|_{H^{1+\varepsilon_{0}(\Omega)}}$.

Second Step.

We take $t_{1} \in\left(0, T_{0}\right)$ so that $v\left(t_{1}\right) \in H_{N}^{2}(\Omega)$ and $u\left(t_{1}\right) \in H^{1}(\Omega)$ and we set $u\left(t_{1}\right)=u_{1}$ and $v\left(t_{1}\right)=v_{1}$. From Theorem 3.1 we allready know that such a time $t_{1}$ exists, arbitrary small. In this step $t$ varies in $\left[t_{1}, T_{0}\right]$. From the first equation of $\left(P_{h}\right)$ we have:

$$
\frac{1}{2} \frac{d}{d t}\|u\|_{L^{2}}^{2}+a\|\nabla u\|_{L^{2}}^{2}+k_{1}\|u\|_{L^{2}}^{2}+q\|u\|_{L^{3}}^{3}=\int_{\Omega} u v d x+\frac{\beta}{2} \int_{\Omega} u^{2} \Delta v d x .
$$

From Young inequality and interpolation inequality (2.1) we get

$$
\begin{aligned}
\int_{\Omega} u^{2} \Delta v d x & \leq \eta\|\Delta v\|_{L^{3}}^{3}+\eta^{-1 / 2}\|u\|_{L^{3}(\Omega)}^{3} \\
& \leq \eta C\|v\|_{H^{3}}^{2}\|v\|_{H^{1}}^{1}+\eta^{-1 / 2}\|u\|_{L^{3}}^{3}, \quad \text { with } \eta>0 \text { arbitrary }
\end{aligned}
$$

Therefore (3.7) together with this yields that

$$
\int_{\Omega} u^{2} \Delta v d x \leq \eta C\|v\|_{H^{3}}^{2}+\eta^{-1 / 2}\|u\|_{L^{3}}^{3},
$$

In addition

$$
\int_{\Omega} u v d x \leq \chi\|u\|_{L^{3}(\Omega)}^{3}+\chi^{-1 / 2}\|v\|_{H^{1}(\Omega)}^{3 / 2}, \quad \text { with } \chi>0 \text { arbitrary . }
$$

Using Biler's Lemma (2.3) we verify from (3.7) that

$$
\|u\|_{L^{3}(\Omega)}^{3} \leq \eta C\|u\|_{H^{1}}^{2}+p\left(\eta^{-1}\right)
$$

with $p$ a positive increasing function, depending on $\|f\|_{L^{\infty}\left(0, T ; L^{2}(\Omega)\right)},\left\|u_{0}\right\|_{L^{2}(\Omega)}$ and $\left\|v_{0}\right\|_{H^{1+\varepsilon_{0}(\Omega)}}$ as well as the constant $C>0$.

Thus we deduce the following inequality

$$
\begin{aligned}
\frac{1}{2} \frac{d}{d t}\|u\|_{L^{2}}^{2}+a\|\nabla u\|_{L^{2}}^{2}+ & k_{1}\|u(t)\|_{L^{2}}^{2}+q\|u\|_{L^{3}}^{3} \\
& \leq \xi\left(\|v\|_{H^{3}}^{2}+\|u\|_{H^{1}}^{2}\right)+p\left(\xi^{-1}\right),
\end{aligned}
$$

with $p$ a positive increasing function depending on $\|f\|_{L^{\infty}\left(0, T ; L^{2}(\Omega)\right)},\left\|u_{0}\right\|_{L^{2}(\Omega)}$ and $\left\|v_{0}\right\|_{H^{1+\varepsilon_{0}(\Omega)}}, \xi>0$ an arbitrary constant.

On the other hand we consider $v$ as a solution of the Cauchy problem

$$
\begin{aligned}
& \frac{d}{d t} v+A_{2} v=k_{1} u+f \quad t_{1} \leq t \leq T_{0} \\
& v\left(t_{1}\right) \in H^{1}(\Omega)
\end{aligned}
$$


in the space $H^{1}(\Omega)$. Since $k_{1} u+f \in L^{2}\left(t_{1}, T_{0} ; H^{1}(\Omega)\right)$ and $v_{1} \in \mathcal{D}\left(A_{2}\right)=$ $H_{N}^{2}(\Omega)$ it follows that $v \in L^{2}\left(t_{1}, T_{0} ; \mathcal{D}\left(A_{2}^{3 / 2}\right) \cap H^{1}\left(t_{1}, T_{0} ; \mathcal{D}\left(A_{2}^{1 / 2}\right)\right.\right.$ and

$$
\frac{d}{d t} A_{2}^{1 / 2} v=-A_{2}^{3 / 2} v+k_{1} A_{2}^{1 / 2} u+A_{2}^{1 / 2} f, \quad t_{1} \leq t \leq T_{0}
$$

Therefore

$$
\frac{d}{d t}\left\|A_{2} v\right\|_{L^{2}}^{2}+\left\|A_{2}^{3 / 2} v\right\|_{L^{2}}^{2} \leq C\left\{\left\|A_{2}^{1 / 2} u\right\|_{L^{2}}^{2}+\left\|A_{2}^{1 / 2} f\right\|_{L^{2}}^{2}\right\} .
$$

As $D\left(A^{\frac{3}{2}}\right) \subset H^{3}(\Omega)$, we obtain

$$
\frac{d}{d t}\left\|A_{2} v\right\|_{L^{2}}^{2}+\delta\|v\|_{H^{3}}^{2} \leq C\left\{\|u\|_{H^{1}}^{2}+\|f\|_{H^{1}}^{2}\right\}
$$

with some $\delta>0$. Let $a_{1}=\min \left(a, k_{1}\right)>0$. We now sum up (3.8) multiplied by $\frac{2 C}{a_{1}}$, where $C>0$ is the constant appearing in (3.9), and (3.9). Then it follows that:

$$
\begin{array}{r}
\frac{d}{d t}\left\{\frac{C}{a_{1}}\|u\|_{L^{2}}^{2}+\left\|A_{2} v\right\|_{L^{2}}^{2}\right\}+C\left(1-\xi \frac{2 C}{a_{1}}\right)\|u\|_{H^{1}}^{2}+\left(\delta-\xi \frac{2 C}{a_{1}}\right)\|v\|_{H^{3}}^{2} \\
\leq C_{1}\left\{\|f(t)\|_{H^{1}}^{2}+p\left(\xi^{-1}\right)\right\},
\end{array}
$$

with some constant $C_{1}>0$ independent of $T_{0}$. Choosing $\xi$ small enough we conclude that:

$\int_{t_{1}}^{s}\left(\|v(t)\|_{H^{3}}^{2}+\|u(t)\|_{H^{1}}^{2}\right) d t \leq C_{2}\left\{\left\|u_{1}\right\|_{L^{2}}^{2}+\left\|v_{1}\right\|_{H^{2}}^{2}+\int_{t_{1}}^{T}\left(\|f(t)\|_{H^{1}}^{2}+1\right) d t\right\}$

with some constant $C_{2}>0$ dependent on $\|f\|_{L^{\infty}\left(0, T ; L^{2}(\Omega)\right)}$ and the initial condition $U_{0}$ through $\left\|u_{0}\right\|_{L^{2}}$ and $\left\|v_{0}\right\|_{H^{1+\varepsilon_{0}}}$, but independent of $T_{0}$. The norms $\|u\|_{L^{2}\left(t_{1}, T_{0} ; H^{1}(\Omega)\right)}$ and $\|v\|_{L^{2}\left(t_{1}, T_{0} ; H^{3}(\Omega)\right)}$ do not depend on $T_{0}$ and hence those of $\|u\|_{C\left(\left[t_{1}, T_{0}\right] ; L^{2}(\Omega)\right)}$ and $\|v\|_{C\left(\left[t_{1}, T_{0}\right] ; H^{2}(\Omega)\right)}$ do not depend either.

In particular this shows that the solution $(u, v)$ can be extended as a weak solution beyond the $T_{0}$.

\subsection{Exponential attractor.}

Let suppose that $f$ is a positive constant function. Then we have the following: 
Proposition 3.5. Let $u_{0} \in H_{N}^{2}(\Omega)$ and $v_{0} \in H_{N}^{3}(\Omega)$ be nonnegative functions. Let $u, v$ be the global solution of $\left(P_{h}\right)$. Then, with some continuous increasing function $p(\cdot)$ the following estimate holds:

$$
\|u(t)\|_{H^{2}(\Omega)}+\|v(t)\|_{H^{3}(\Omega)} \leq p\left(\left\|u_{0}\right\|_{H^{2}(\Omega)}+\left\|v_{0}\right\|_{H^{3}(\Omega)}+f\right),
$$

for $0<t<\infty$.

Proof. Using (3.10) we deduce the existence of two constants $\sigma>0$ and $C>0$ such that

$$
\begin{aligned}
\|u(t)\|_{L^{2}}^{2}+\|v(t)\|_{H^{2}}^{2} \leq C e^{-\sigma t}\left(\left\|u_{0}\right\|_{L^{2}}^{2}\right. & \left.+\left\|v_{0}\right\|_{H^{2}}^{2}\right) \\
& +p\left(f+N_{l o g}^{1}\left(u_{0}\right)+\left\|v_{0}\right\|_{H^{1}}\right)
\end{aligned}
$$

Multiplying the first equation of $\left(P_{h}\right)$ by $\Delta u$ and integrating over $\Omega$, gives

$$
\begin{aligned}
& \frac{1}{2} \frac{d}{d t}\|\nabla u\|_{L^{2}}^{2}+a\|\Delta u\|_{L^{2}}^{2}+k_{1}\|\nabla u\|_{L^{2}}^{2} \\
& \leq \beta\left(\varepsilon\|\Delta u\|_{L^{2}}^{2}+\frac{1}{2 \varepsilon} \int_{\Omega}|\nabla u|^{2}|\nabla v|^{2} d x+\frac{1}{2 \varepsilon} \int_{\Omega}|u|^{2}|\Delta v|^{2} d x\right) \\
& \quad+\varepsilon^{\prime}\|\nabla u\|_{L^{2}}^{2}+C_{\varepsilon^{\prime}}\|\nabla v\|_{L^{2}}^{2},
\end{aligned}
$$

where $\varepsilon, \varepsilon^{\prime}$ and $C_{\varepsilon^{\prime}}$ are positive constants derived from Young inequality. Using technical inequalities proved in ([13] proposition 4.1) we obtain

$$
\begin{aligned}
& \frac{1}{2} \frac{d}{d t}\|\nabla u\|_{L^{2}}^{2}+(a-\beta \varepsilon)\|\Delta u\|_{L^{2}}^{2}+\left(k_{1}-\varepsilon^{\prime}\right)\|\nabla u\|_{L^{2}}^{2} \\
& \leq \frac{\beta}{2 \varepsilon}\left(\int_{\Omega}|\nabla u|^{2}|\nabla v|^{2} d x+\int_{\Omega}|u|^{2}|\Delta v|^{2} d x\right)+C_{\varepsilon^{\prime}}\|\nabla v(t)\|_{L^{2}}^{2} \\
& \quad \leq \frac{\beta}{2 \varepsilon}\left(\eta\|\Delta u\|_{L^{2}}^{2}+p\left(\|u\|_{L^{2}}+\|v\|_{H^{2}}+\eta^{-1}\right)\right)+C_{\varepsilon^{\prime}}\|\nabla v\|_{L^{2}}^{2} .
\end{aligned}
$$

Taking $\eta=\varepsilon^{2}, \varepsilon=\frac{a}{2 \beta}$ leads to

$$
\begin{aligned}
\frac{d}{d t}\|\nabla u\|_{L^{2}}^{2}+a\|\Delta u\|_{L^{2}}^{2}+ & 2\left(k_{1}-\varepsilon^{\prime}\right)\|\nabla u\|_{L^{2}}^{2} \\
& \leq \frac{\beta^{2}}{a} p\left(\|u\|_{L^{2}}+\|v\|_{H^{2}(\Omega)}\right)+C_{\varepsilon^{\prime}}\|\nabla v\|_{L^{2}}^{2} .
\end{aligned}
$$

Take the second equation of $\left(P_{h}\right)$ operated by $\Delta$, choose $\Delta^{2} v$ as a test function and integrate the product in $\Omega$. After some calculations as in [13] we have

$$
\frac{d}{d t}\|\nabla \Delta v\|_{L^{2}}^{2}+d\left\|\Delta^{2} v\right\|_{\left.L^{2}\right)}^{2}+2 k_{2}\|\nabla \Delta v\|_{L^{2}}^{2} \leq \frac{k_{1}^{2}}{d}\|\Delta u\|_{L^{2}}^{2} .
$$


We sum (3.14) multiplied by $\gamma$ and (3.13). Thus we obtain:

$$
\begin{aligned}
\frac{d}{d t}\left(\|\nabla u\|_{L^{2}}^{2}+\gamma\|\nabla \Delta v\|_{L^{2}}^{2}\right)+\gamma d\left\|\Delta^{2} v\right\|_{L^{2}}^{2}+\left(a-\frac{\gamma k_{1}^{2}}{d}\right)\|\Delta u\|_{L^{2}}^{2} \\
\quad+2\left(k_{1}-\varepsilon^{\prime}\right)\left(\|\nabla u\|_{L^{2}}^{2}+\frac{k_{2} \gamma}{k_{1}-\varepsilon^{\prime}}\|\nabla \Delta v\|_{L^{2}}^{2}\right) \leq p\left(\|u\|_{L^{2}}+\|v\|_{H^{2}}\right) .
\end{aligned}
$$

Then for $\gamma$ and $\varepsilon^{\prime}$ small enough, there exists a positive constant $\sigma^{\prime}$ such that

$$
\begin{aligned}
\frac{d}{d t}\left(\|\nabla u\|_{L^{2}}^{2}+\gamma\|\nabla \Delta v\|_{L^{2}}^{2}\right)+\sigma^{\prime}\left(\|\nabla u\|_{L^{2}}^{2}+\gamma\|\nabla \Delta v(t)\|_{L^{2}}^{2}\right) & \\
& \leq p\left(\|u\|_{L^{2}}+\|v\|_{H^{2}}\right) .
\end{aligned}
$$

So, we can find $\chi>0$ such as (3.11) is valid when $\sigma=\chi$ and

$$
\begin{aligned}
\|u(t)\|_{H^{1}(\Omega)}^{2}+\|v(t)\|_{H^{3}}^{2} \leq e^{-\chi t}\left(\left\|u_{0}\right\|_{H^{1}}^{2}\right. & \left.+\left\|v_{0}\right\|_{H^{3}}^{2}\right) \\
& +p\left(f+\left\|u_{0}\right\|_{L^{2}}+\left\|v_{0}\right\|_{H^{2}}\right) .
\end{aligned}
$$

We verify also that

$$
\begin{aligned}
\int_{0}^{t}\left(\left\|\Delta^{2} v(s)\right\|_{L^{2}}^{2}+\|u(s)\|_{H^{2}}^{2}\right) d s \leq C\left(\left\|v_{0}\right\|_{H^{3}}^{2}\right. & \left.+\left\|u_{0}\right\|_{H^{1}}^{2}\right) \\
& +t p\left(f+\left\|u_{0}\right\|_{L^{2}}+\left\|v_{0}\right\|_{H^{2}}\right) .
\end{aligned}
$$

Finally, taking the first equation of $\left(P_{h}\right)$ operated by $\nabla$ and multiplied by $\nabla \Delta u$, gives as in $[13]$

$$
\begin{aligned}
& \frac{1}{2} \frac{d}{d t}\|\Delta u\|_{L^{2}}^{2}+a\|\nabla \Delta u\|_{L^{2}}^{2}=\beta \int_{\Omega} \nabla(\nabla \cdot u \nabla v) \cdot \nabla \Delta u d x \\
& \quad+k_{1} \int_{\Omega} \nabla u \cdot \nabla \Delta u d x+2 q \int_{\Omega} u \nabla u \cdot \nabla \Delta u d x-k_{2} \int_{\Omega} \nabla v \cdot \nabla \Delta u d x
\end{aligned}
$$

that is

$$
\begin{aligned}
& \frac{1}{2} \frac{d}{d t}\|\Delta u\|_{L^{2}(\Omega)}^{2}+a\|\nabla \Delta u\|_{L^{2}(\Omega)}^{2} \leq \frac{a}{2}\|\nabla \Delta u\|_{L^{2}}^{2} \\
& \quad+C \int_{\Omega} \mid \nabla\left(\left.\nabla \cdot(u \nabla v)\right|^{2} d x+C\left(\int_{\Omega}|u \nabla u|^{2} d x+\|\nabla v\|_{L^{2}}^{2}\right) .\right.
\end{aligned}
$$

The terms $\int_{\Omega} \mid \nabla\left(\left.\nabla \cdot(u \nabla v)\right|^{2} d x\right.$ and $\int_{\Omega}|u \nabla u|^{2} d x$ of (3.18) can be estimated (see [13], proof of proposition 4.1, step 6) by

$$
\eta\|\nabla \Delta u\|_{L^{2}}^{2}+p\left(\|u\|_{H^{1}}+\|v\|_{H^{3}},+\eta^{-1}\right)
$$


With an arbitrary $\eta>0$. Thus we obtain:

$$
\begin{aligned}
\frac{1}{2} \frac{d}{d t}\|\Delta u\|_{L^{2}}^{2}+\frac{a}{2}\|\nabla \Delta u\|_{L^{2}}^{2} & +\zeta\|\Delta u\|_{L^{2}}^{2} \\
& \leq \eta\|\nabla \Delta u\|_{L^{2}}^{2}+p\left(\|u\|_{H^{1}}+\|v\|_{H^{3}}+\eta^{-1}\right) .
\end{aligned}
$$

Hence we can find a constant $\chi>0$ such that (3.16) is valid and

$$
\|u(t)\|_{H^{2}}^{2} \leq e^{-\chi t}\left\|u_{0}\right\|_{H^{2}}^{2}+p\left(f+\left\|u_{0}\right\|_{H^{1}}+\left\|v_{0}\right\|_{H^{3}}\right) .
$$

To prove the existence of an exponential attractor, we will use the following result:

Proposition 3.6. Let $u_{0} \in L^{2}(\Omega), v_{0} \in H^{1+\varepsilon_{0}}(\Omega)$ be nonnegative functions. Then there exists a continuous increasing function $p(\cdot)$, independent of $u_{0}$ and $v_{0}$ such that

$$
\|u(t)\|_{H^{2}}^{2}+\|v(t)\|_{H^{3}}^{2} \leq p\left(f+N_{l o g}^{1}\left(u_{0}\right)+\left\|v_{0}\right\|_{H^{1}(\Omega)}+t^{-1}\right)
$$

Proof. Since the proof follows exactly the same ideas and technical difficulties as in the proof of Theorem 4.6 [13] we skip it here.

We can now prove the existence of an exponential attractor: Let $H=$ $L^{2}(\Omega) \times H^{1}(\Omega)$ and consider the initial value problem

$$
\begin{aligned}
& \frac{d U}{d t}+A U=G(U) \\
& U(0)=U_{0}
\end{aligned}
$$

in $H$, with $A$ as in section 3.1 and $D(A)=H_{n}^{2}(\Omega) \times H_{n}^{3}(\Omega)$ and

$$
G(U):=\left(\begin{array}{c}
\beta \nabla(u \nabla v)-q|u| u+k_{2} v \\
k_{1} u+f
\end{array}\right)
$$

Let $K=\left\{(u, v) \in L_{+}^{2}(\Omega) \times H_{+}^{1+\varepsilon_{0}}(\Omega)\right\}$ be the space of initial values and $U_{0} \in K$.

We proved already the existence of a unique global solution $U=(u, v)$ continuous with respect to the initial condition $U_{0}$. We define then a continuous semigroup $\left\{S(t)_{t \geq 0}\right\}$ on $K$ by $S(t) U_{0}=U(t)$. For a fixed $t>0, S(t)$ maps $K$ into $K \cap D(A)$.

Let denote $B_{r}:=\left\{(u, v) \in K ;\left\|u_{0}\right\|_{L^{2}}+\left\|v_{0}\right\|_{H^{1+\varepsilon_{0}}} \leq r\right\}$ a bounded ball of $K$ with radius $r>0$. 
Proposition 3.7. There exists a universal constant $C>0$ such that the following statement holds: for each $r>0$ there exists a time $t_{r}>0$ such that

$$
\sup _{t \geq t_{r}} \sup _{U_{0} \in B_{r}}\left\|S(t) U_{0}\right\|_{H^{2}(\Omega) \times H^{3}(\Omega)} \leq C
$$

Proof. Fix $0<r<\infty$. By $t_{r}$ and $C_{r}$ we shall denote some time and positive constant which depend on $r$ but are uniform in $U_{0} \in B_{r}$, respectively. By the Proposition 3.6, there exist a time $t_{r}$ and a constant $C_{r}$ such that for $t \geq t_{r}$

$$
\|u(t)\|_{H^{2}}^{2}+\|v(t)\|_{H^{3}}^{2} \leq C_{r}
$$

The desired estimate will be established step by step.

Let us add the first equation of $\left(P_{h}\right)$ and the second one multiplied by 2 and let us integrate in space the result. If $\Phi(t):=\|u(t)\|_{L^{1}}+2\|v(t)\|_{L^{1}}$ we obtain:

$\frac{d}{d t} \Phi(t)+\frac{k_{2}}{2} \Phi(t)=\int_{\Omega}\left(-q u^{2}+k_{1} u+\frac{k_{2}}{2} u\right) d x+f|\Omega| \leq\left\{\frac{1}{4 q}\left(k_{1}+\frac{k_{2}}{2}\right)^{2}+f\right\}|\Omega|$.

Thus

$\Phi(t) \leq\left\{\Phi(0)-\frac{2}{k_{2}}\left(\frac{1}{4 q}\left(k_{1}+\frac{k_{2}}{2}\right)^{2}+f\right)|\Omega|\right\} e^{-\frac{k_{2}}{2} t}+\frac{2}{k_{2}}\left(\frac{1}{4 q}\left(k_{1}+\frac{k_{2}}{2}\right)^{2}+f\right)|\Omega|$,

and we deduce

$$
\|u(t)\|_{L^{1}}+2\|v(t)\|_{L^{1}} \leq C\left(C_{r} e^{-c t}+1\right),
$$

with $C, c>0$ universal constants and $C_{r}>0$ a constant depending in $r$. This shows that there exists a time denoted by $t_{r}$ such that for all $t \geq t_{r}$

$$
\|u(t)\|_{L^{1}}+\|v(t)\|_{L^{1}} \leq C
$$

with $C>0$ a universal constant.

From (3.6) and (3.22) it follows that

$$
g(t) \leq(g(0)-C) e^{-\sigma\left(t-t_{r}\right)}+C \text { for } t \geq t_{r} .
$$

Then there exists another time $t_{r}$ and another universal constant $C>0$ such that

$$
\|v(t)\|_{H^{1}} \leq C \text { and } N_{l o g}^{1}(u(t)) \leq C \text { for } t \geq t_{r} .
$$


From (3.11) and (3.21) we deduce that

$$
\|v(t)\|_{H^{2}(\Omega)}+\|u(t)\|_{L^{2}(\Omega)} \leq C_{r} e^{-\sigma\left(t-t_{r}\right)}+C \text { for } t \geq t_{r} .
$$

and that there exist another time $t_{r}$ and another constant $C>0$, such that

$$
\|v(t)\|_{H^{2}(\Omega)}+\|u(t)\|_{L^{2}(\Omega)} \leq C \text { for } t \geq t_{r}
$$

Finally using (3.20), (3.16) and repeat the argument we finish the proof.

Let $\mathscr{B}=\left\{(u, v) \in H_{N}^{2}(\Omega) \times H_{N}^{3}(\Omega) /\|u\|_{H^{2}(\Omega)}+\|v\|_{H^{3}(\Omega)} \leq C\right\} \cap K$ with $\mathrm{C}$ the constant appearing in proposition 3.7. We proved that $\mathscr{B}$ is a compact absorbing set for $\left(\{S(t)\}_{t \geq 0}, K\right)$. Hence by Temam $([19])$, there exist a global attractor $\mathscr{A} \subset K$, where $\mathscr{A}$ is a compact and connected subset of $K$.

Let $\mathscr{H}=\overline{\cup_{t \geq t_{\mathscr{B}}} S(t) \mathscr{B}}$ where $t_{\mathscr{B}}$ is such that $S(t) \mathscr{B} \subset \mathscr{B}$. Then $\mathscr{H}$ is a compact set of $K$ with $\mathscr{A} \subset \mathscr{H} \subset K$. Since $\mathscr{H}$ is absorbing and positively invariant for $\left\{S(t)_{t \geq 0}\right\}$ we apply to the dynamical system $\left(\{S(t)\}_{t \geq 0}, \mathscr{H}\right)$ the following

Theorem. (Theorem 3.1 [20])

Let $\Gamma\left(t, U_{0}\right)=S(t) U_{0}$ be a mapping from $[0, T] \times \mathscr{H}$ into $\mathscr{H}$. If $G$ satisfies

$$
\|G(U)-G(V)\| \leq\left\|A^{\frac{1}{2}}(U-V)\right\|, U, V \in \mathscr{H}
$$

and $\Gamma$ is such that

$$
\left\|\Gamma\left(t, U_{0}\right)-\Gamma\left(s, V_{0}\right)\right\| \leq C_{T}\left(|t-s|+\left\|U_{0}-V_{0}\right\|_{H}, t, s \in[0, T], U_{0}, V_{0} \in \mathscr{H}\left(C_{2}\right)\right.
$$

for each $T>0$, then there is an exponential attractor $\mathscr{M}$ for $(\{S(t)\}, \mathscr{H})$.

Thus we obtain

Theorem 3.8. There exists an exponential attractor $\mathscr{M}$ of the dynamical system $\left(\{S(t)\}_{t \geq 0}, \mathscr{H}\right)$ in $H$

Proof. Since the forcing term $f$ is constant and the reaction coupling of the first equation of (E) is linear in $U: k_{2} v$, the proof is the same as provided in ([13], Theorem 5.1). 


\section{Second case: $h(u)=u(M-u)$.}

Let $M$ be a positive constant and consider a continuous function $\tilde{h}$ of $h$ such as

$$
\left\{\begin{array}{l}
\tilde{h}(u)=u(M-u) \text { if } 0 \leq u \leq M \\
\tilde{h}(u)=0 \text { otherwise. }
\end{array}\right.
$$

Then we have the following:

Proposition 4.1. Let $\varepsilon_{0}>0$ and $f$ be a nonnegative function in $L^{2}\left(0, T ; H^{1}(\Omega)\right) \cap$ $E^{\infty}\left(\Omega_{T}\right)$. For each nonnegative initial condition $\left(u_{0}, v_{0}\right)$ in $L^{2}(\Omega) \times H^{1+\varepsilon_{0}}(\Omega)$ there exists a constant $T_{0}$ such that $0<T_{0} \leq T$ and a unique nonnegative solution $(u, v)$ of $\left(P_{\tilde{h}}\right)$ such that:

$$
\begin{aligned}
& u \in H^{1}\left(0, T_{0} ;\left(H^{1}(\Omega)\right)^{\prime}\right) \cap C\left(\left[0, T_{0}\right] ; L^{2}(\Omega)\right) \cap L^{2}\left(0, T_{0} ; H^{1}(\Omega)\right) \\
& v \in H^{1}\left(0, T_{0} ; H^{\varepsilon_{0}}(\Omega)\right) \cap C\left(\left[0, T_{0}\right] ; H^{1+\varepsilon_{0}}(\Omega)\right) \cap L^{2}\left(0, T_{0} ; H_{\nu}^{2+\varepsilon_{0}}(\Omega)\right)
\end{aligned}
$$

Proof. The proof is essentially the same as in section 3.2 .

Moreover we can prove the following:

Lemma 4.2. Let suppose that $M \geq\left(\frac{\|f\|_{L^{\infty}\left(\Omega_{T}\right)}}{q}\right)^{\frac{1}{2}}$ and $M^{\prime}=\frac{q M^{2}+k_{1} M}{k_{2}}>0$. If the initial condition $\left(u_{0}, v_{0}\right)$ satisfies almost everywhere in $\Omega$ the following inequalities:

$$
0 \leq u_{0}(x) \leq M, \quad 0 \leq v_{0}(x) \leq M^{\prime},
$$

then the solution $(u, v)$ of $\left(P_{\tilde{h}}\right)$ satisfies:

$$
0 \leq u(t, x) \leq M \quad 0 \leq v(t, x) \leq M^{\prime},
$$

almost everywhere in $\Omega_{T}$.

Proof. Let define $\tilde{u}=M-u$ and $\tilde{v}=M^{\prime}-v$. Thus we get:

$$
\begin{aligned}
& \tilde{u}_{t}=a \Delta \tilde{u}-\beta \operatorname{div}(\tilde{h}(\tilde{u}) \nabla \tilde{v})-\left(2 q M+k_{1}\right) \tilde{u}+q \tilde{u}^{2}+k_{2} \tilde{v}+q M^{2}+k_{1} M-k_{2} M^{\prime} \\
& \tilde{v}_{t}=d \Delta \tilde{v}-k_{2} \tilde{v}+k_{1} \tilde{u}+k_{2} M^{\prime}-k_{1} M-f
\end{aligned}
$$

As $M \geq\left(\frac{\|f\|_{L^{\infty}\left(\Omega_{T}\right)}}{q}\right)^{\frac{1}{2}}$, and $M^{\prime}=\frac{q M^{2}+k_{1} M}{k_{2}}$ we obtain

$$
q M^{2}+k_{1} M-k_{2} M^{\prime}=0
$$

and

$$
k_{2} M^{\prime}-k_{1} M-f \geq 0 .
$$


We multiply the first equation by $-\tilde{u}^{-}$and the second by $-\tilde{v}^{-}$and we integrate in space. Thank to the identity: $\int_{\Omega} \tilde{h}(\tilde{u}) \nabla \tilde{v} \nabla \tilde{u}^{-} d x=0$ and since $q \tilde{u}^{2} \tilde{u}^{-}, k_{2} \tilde{u}^{-} \tilde{v}^{+}, k_{1} \tilde{v}^{-} \tilde{u}^{+} \geq 0$ almost everywhere in $\Omega$, we deduce:

$$
\frac{1}{2} \frac{d}{d t}\left\|\tilde{u}^{-}\right\|_{L^{2}(\Omega)}^{2} \leq\left(2 q M+k_{1}\right)\left\|\tilde{u}^{-}\right\|_{L^{2}(\Omega)}^{2}+k_{2} \int_{\Omega} \tilde{v}^{-} \tilde{u}^{-} d x
$$

and

$$
\frac{1}{2} \frac{d}{d t}\left\|\tilde{v}^{-}\right\|_{L^{2}(\Omega)}^{2} \leq k_{2}\left\|\tilde{v}^{-}\right\|_{L^{2}(\Omega)}^{2}+k_{1} \int_{\Omega} \tilde{v}^{-} \tilde{u}^{-} d x .
$$

Taking the sum of the two previous inequalities and using Young inequality it follows:

$$
\frac{d}{d t}\left(\left\|\tilde{u}^{-}\right\|_{L^{2}(\Omega)}^{2}+\left\|\tilde{v}^{-}\right\|_{L^{2}(\Omega)}^{2}\right) \leq C\left(\left\|\tilde{u}^{-}\right\|_{L^{2}(\Omega)}^{2}+\left\|\tilde{v}^{-}\right\|_{L^{2}(\Omega)}^{2}\right)
$$

for some constant $C>0$. By Gronwall lemma we get

$$
\left\|\tilde{u}^{-}\right\|_{L^{2}(\Omega)}^{2}+\left\|\tilde{v}^{-}\right\|_{L^{2}(\Omega)}^{2}=0 .
$$

which completes the proof.

Remark. i) If the hypothesis of Lemma 4.2 are fulfilled, thanks to this Lemma, the solution obtained in proposition 4.1 is global in $\Omega_{T}$,

ii) By Proposition 4.1 and Lemma 4.2 it follows that $(u, v)$ is also a solution of $\left(P_{h}\right)$, with $h(u)=u(M-u)$.

The uniqueness of the solution is obtained in the following

Theorem 4.3. Let $f \in L^{\infty}\left(\Omega_{T}\right) \cap L^{2}\left(0, T ; H^{1}(\Omega)\right)$ be a nonnegative function. Let $h(u)=u(M-u)$ and suppose that $M \geq\left(\frac{\left(\|f\|_{L^{\infty}\left(\Omega_{T}\right)}\right.}{q}\right)^{\frac{1}{2}}$. Let $\left(u_{0}, v_{0}\right) \in L^{2}(\Omega) \times H^{1+\varepsilon_{0}}(\Omega)$ such that $0 \leq u_{0} \leq M$ and $0 \leq v_{0} \leq M^{\prime}$ with $M^{\prime}=\frac{q M^{2}+k_{1} M}{k_{2}}$. Then there exists a unique global solution for $\left(P_{h}\right)$ which is nonnegative and such that

$$
\begin{aligned}
& u \in L^{\infty}\left(\Omega_{T}\right) \cap H^{1}\left(0, T ;\left(H^{1}(\Omega)\right)^{\prime}\right) \cap C\left([0, T] ; L^{2}(\Omega)\right) \cap L^{2}\left(0, T ; H^{1}(\Omega)\right) \\
& v \in H^{1}\left(0, T ; H^{\varepsilon_{0}}(\Omega)\right) \cap C\left([0, T] ; H^{1+\varepsilon_{0}}(\Omega)\right) \cap L^{2}\left(0, T ; H^{2+\varepsilon_{0}}(\Omega)\right)
\end{aligned}
$$

and

$$
0 \leq u \leq M \quad 0 \leq v \leq M^{\prime}
$$

Proof. We skip here the proof of uniqueness since there is rigorously the same as in Theorem 5.3. 


\section{A three dimensional domain.}

In order to prove the global existence of a solution of system $\left(P_{h}\right)$, we supposed in the previous sections that $\Omega$ was a two dimensional domain and the initial conditions $\left(u_{0}, v_{0}\right) \in L^{\infty}(\Omega) \times H^{1+\varepsilon_{0}}(\Omega)$ were nonnegative and verifying some regularity conditions. These conditions are quite restrictive for a model of soil organic carbon and three dimensional domains are obviously more relevant in applications than bidimensional ones.

In this section we prove that if $\Omega$ is of dimension less than or equal to 3 , if $h=\tilde{h}$ (4.1) and if both initial conditions and forcing term are nonnegative and less regular that in the previous section : $\left(u_{0}, v_{0}\right) \in\left(L^{2}(\Omega)\right)^{2}$ and $f \in L^{2}\left(0, T ; L^{2}(\Omega)\right)$, then the system $\left(P_{h}\right)$ has a global nonnegative solution. Furthermore, if $\left(u_{0}, v_{0}\right) \in\left(L^{\infty}(\Omega)\right)^{2}$ and $f \in L^{\infty}\left(\Omega_{T}\right)$, then the solution is unique.

Here we use the following setting:

$$
\begin{aligned}
& V=H^{1}(\Omega) \times H^{1}(\Omega), \\
& H=L^{2}(\Omega) \times L^{2}(\Omega), \\
& V^{\prime}=\left(H^{1}(\Omega)\right)^{\prime} \times\left(H^{1}(\Omega)\right)^{\prime} .
\end{aligned}
$$

We let $\tilde{h}$ be the continuous function defined by (4.1). Let us consider the following system:

$$
\left\{\begin{array}{l}
\partial_{t} u-a \Delta u=-\beta \operatorname{div}(\tilde{h}(\bar{u}) \nabla v)-k_{1} u-q|u| u+k_{2} v \quad \text { in } \Omega_{T} \\
\partial_{t} v-d \Delta v=-k_{2} v+k_{1} u+f \quad \text { in } \Omega_{T} \\
\nabla u \cdot \eta(x)=\nabla v \cdot \eta(x)=0 \quad \text { on } \Sigma_{T} \\
u(0, \cdot)=u_{0}, v(0, \cdot)=v_{0} \quad \text { in } \Omega
\end{array}\right.
$$

where $\left(u_{0}, v_{0}\right) \in\left(L^{2}(\Omega)\right)^{2}, f \in L^{2}\left(0, T ; L^{2}(\Omega)\right)$ and $\bar{u}$ is a function in $X=L^{2}\left(\Omega_{T}\right)$.

For the sake of simplicity we take $\operatorname{dim}(\Omega)=3$. since all results remain the same if $\operatorname{dim}(\Omega)<3$.

We will apply the Schauder fixed point theorem but let us first gather some more information.

First Step: Invariant Ball

For any function $\bar{u} \in X$ the existence of a unique local solution of (P-S) $\left(u_{\bar{u}}, v_{\bar{u}}\right)$ follows by direct application of Theorem 2.1. Additionally we have the following: 
Proposition 5.1. Let $\left(u_{0}, v_{0}\right) \in\left(L^{2}(\Omega)\right)^{2}$ and $f \in L^{2}\left(0, T ; L^{2}(\Omega)\right)$.

1. For any $\bar{u} \in X$ the unique local solution $\left(u_{\bar{u}}, v_{\bar{u}}\right)$ of $(\mathrm{P}-\mathrm{S})$ is global and satisfies:

$$
\begin{aligned}
& u_{\bar{u}} \in H^{1}\left(0, T ;\left(H^{1}(\Omega)\right)^{\prime}\right) \cap C\left([0, T] ; L^{2}(\Omega)\right) \cap L^{2}\left(0, T ; H^{1}(\Omega)\right), \\
& v_{\bar{u}} \in H^{1}\left(0, T ;\left(H^{1}(\Omega)\right)^{\prime}\right) \cap C\left([0, T] ; L^{2}(\Omega)\right) \cap L^{2}\left(0, T ; H^{1}(\Omega)\right) .
\end{aligned}
$$

2. Furthermore, for all $\bar{u} \in X$, there exist two constant $R>0$ and $C>0$ such that:

$$
\left\|u_{\bar{u}}\right\|_{L^{2}\left(\Omega_{T}\right)} \leq R \quad \text { and }\|u\|_{W} \leq C
$$

where

$$
W=\left\{u \in L^{2}\left(0, T ; H^{1}(\Omega)\right), u_{t} \in L^{2}\left(0, T ;\left(H^{1}(\Omega)\right)^{\prime}\right)\right\}
$$

Proof. To prove that $\left(u_{\bar{u}}, v_{\bar{u}}\right)$ is global in time, we multiply the first equation by $u_{\bar{u}}$ and the second by $v_{\bar{u}}$ and use Young inequality to get

$$
\begin{gathered}
\frac{1}{2} \frac{d}{d t}\left\|u_{\bar{u}}\right\|_{L^{2}(\Omega)}^{2}+ \\
a\left\|\nabla u_{\bar{u}}\right\|_{L^{2}(\Omega)}^{2}+\int_{\Omega}\left\{k_{1}\left|u_{\bar{u}}\right|^{2}+q\left|u_{\bar{u}}\right|^{3}\right\} \leq k_{2} \int_{\Omega}\left\{u_{\bar{u}}^{2}+\frac{1}{4} v_{\bar{u}}^{2}\right\} d x \\
+\frac{M_{\bar{u}}^{2}}{4}\left(\frac{M^{2}}{8 a}\left\|\nabla v_{\bar{u}}\right\|_{L^{2}(\Omega)}^{2}+\frac{a}{2} \frac{4}{M^{2}}\left\|\nabla u_{\bar{u}}\right\|_{L^{2}(\Omega)}^{2}\right),
\end{gathered}
$$

and

$$
\begin{aligned}
\frac{1}{2} \frac{d}{d t}\left\|v_{\bar{u}}\right\|_{L^{2}(\Omega)}^{2}+ & d\left\|\nabla v_{\bar{u}}\right\|_{L^{2}(\Omega)}^{2}+k_{2}\left\|v_{\bar{u}}\right\|_{L^{2}(\Omega)}^{2} \leq \frac{k_{2}}{2}\left\|v_{\bar{u}}\right\|_{L^{2}(\Omega)}^{2} \\
& +\int_{\Omega}\left\{\frac{k_{1}^{2}}{2 k_{2}} u_{\bar{u}}^{2}\right\} d x+\frac{k_{2}}{4}\left\|v_{\bar{u}}\right\|_{L^{2}(\Omega)}^{2}+\frac{1}{k_{2}}\|f\|_{L^{2}(\Omega)}^{2} .
\end{aligned}
$$

Multiplying by $\rho>0$ the first inequality and adding to the second one gives:

$$
\begin{aligned}
& \frac{1}{2} \frac{d}{d t}\left(\rho\left\|u_{\bar{u}}\right\|_{L^{2}(\Omega)}^{2}+\left\|v_{\bar{u}}\right\|_{L^{2}(\Omega)}^{2}\right)+\frac{\rho a}{2}\left\|\nabla u_{\bar{u}}\right\|_{L^{2}(\Omega)}^{2}+d\left\|\nabla v_{\bar{u}}\right\|_{L^{2}(\Omega)}^{2} \\
& +\int_{\Omega}\left\{\rho k_{1}\left|u_{\bar{u}}\right|^{2}+\rho q\left|u_{\bar{u}}\right|^{3}\right\} \leq \rho \frac{M^{4}}{32 a}\left\|\nabla v_{\bar{u}}\right\|_{L^{2}(\Omega)}^{2}+C \int_{\Omega}\left|u_{\bar{u}}\right|^{2}+\frac{1}{k_{2}}\|f\|_{L^{2}(\Omega)}^{2},
\end{aligned}
$$

where $C=\frac{k_{1}^{2}}{2 k_{2}}+k_{2}$. For $\rho=\frac{16 a d}{M^{4}}$ we obtain the following inequality:

$$
\begin{aligned}
\frac{1}{2} \frac{d}{d t}\left(\rho\left\|u_{\bar{u}}\right\|_{L^{2}}^{2}\right. & \left.+\left\|v_{\bar{u}}\right\|_{L^{2}(\Omega)}^{2}\right)+\frac{\rho a}{2}\left\|\nabla u_{\bar{u}}\right\|_{L^{2}(\Omega)}^{2}+\frac{d}{2}\left\|\nabla v_{\bar{u}}\right\|_{L^{2}(\Omega)}^{2} \\
& +\int_{\Omega}\left\{\left(\rho k_{1}-C\right)\left|u_{\bar{u}}\right|^{2}+\rho q\left|u_{\bar{u}}\right|^{3}\right\} \leq \frac{1}{k_{2}}\|f\|_{L^{2}(\Omega)}^{2} .
\end{aligned}
$$


If $\left(\rho k_{1}-C\right) \geq 0$ we finished the proof of part 1 . If $\left(\rho k_{1}-C\right)<0$ then, for any $0<\lambda<\rho q$, let note $K_{\lambda}=\frac{4}{27} \frac{\left(\rho k_{1}-C\right)^{3}}{(\rho q-\lambda)^{2}}<0$. By a simple real analysis argument, we deduce :

$$
\left(\rho k_{1}-C\right)|u|^{2}+\rho q|u|^{3} \geq \lambda|u|^{3}+K_{\lambda},
$$

for any $u \in X$. Hence the inequality (5.2) becomes:

$$
\begin{aligned}
\frac{1}{2} \frac{d}{d t}\left(\rho\left\|u_{\bar{u}}\right\|_{L^{2}(\Omega)}^{2}+\left\|v_{\bar{u}}\right\|_{L^{2}(\Omega)}^{2}\right) & +\frac{\rho a}{2}\left\|\nabla u_{\bar{u}}\right\|_{L^{2}(\Omega)}^{2}+\frac{d}{2}\left\|\nabla v_{\bar{u}}\right\|_{L^{2}(\Omega)}^{2} \\
& +\int_{\Omega}\left\{\lambda\left|u_{\bar{u}}\right|^{3}+K_{\lambda}\right\} \leq \frac{1}{k_{2}}\|f\|_{L^{2}(\Omega)}^{2} .
\end{aligned}
$$

Since $u_{0}, v_{0} \in L^{2}(\Omega)$ we deduce that $u_{\bar{u}}, v_{\bar{u}}$ are bounded in $L^{\infty}\left(0, T ; L^{2}(\Omega)\right) \cap$ $L^{2}\left(0, T, H^{1}(\Omega)\right)$, and this bound does not depend on $\bar{u}$. Using interpolation technique we obtain that $u_{\bar{u}}$ is bounded in $\in L^{4}\left(0, T ;\left(L^{3}(\Omega)\right)\right.$ and consequently $\left|u_{\bar{u}}\right| u_{\bar{u}}$ is bounded in $L^{2}\left(0, T ;\left(L^{\frac{3}{2}}(\Omega)\right)\right.$, independent of $\bar{u}$.

Combining Hölder inequality, the boundedness of $u_{\bar{u}}, v_{\bar{u}}$ in $L^{2}\left(0, T, H^{1}(\Omega)\right)$ and $L^{4}\left(0, T ;\left(L^{3}(\Omega)\right)\right.$ and the continous injection of $L^{2}\left(0, T ; H^{1}(\Omega)\right)$ into $L^{2}\left(0, T ;\left(L^{3}(\Omega)\right)\right.$ we obtain that $\partial_{t} u_{\bar{u}}, \partial_{t} v_{\bar{u}}$ are bounded in $L^{2}\left(0, T ;\left(H^{1}(\Omega)\right)^{\prime}\right)$, independent of $\bar{u}$. So we finish the proof.

We can then define the mapping $\Pi: X \rightarrow X$ such that $u_{\bar{u}}=\Pi(\bar{u})$ is the unique solution of (P-S). From (5.1) the ball $B_{R} \subset X$ is invariant by $\Pi$.

Second Step: Compactness of $\Pi\left(B_{R}\right)$.

The second statement of the previous proposition implies that $\Pi\left(B_{R}\right) \subset$ $\left\{u \in W,\|u\|_{W} \leq C\right\}$. But the embedding of $W$ into $L^{2}\left(0, T, L^{2}(\Omega)\right)$ is compact thanks to the Aubin-Lions lemma.

Third Step: $\Pi$ is a continuous mapping.

Let $z_{n} \in B_{R}$ such that $z_{n} \rightarrow z$ in $L^{2}\left(\Omega_{T}\right)$ strong and let $u_{n}=\Pi\left(z_{n}\right)$. Then $U_{n}=\left(u_{n}, v_{n}\right)$ satisfies the system $(\mathrm{P}-\mathrm{S})_{n}$ :

$$
\left\{\begin{array}{l}
\partial_{t} u_{n}-a \Delta u_{n}=-\beta \operatorname{div}\left(\tilde{h}\left(z_{n}\right) \nabla v_{n}\right)-k_{1} u_{n}-q\left|u_{n}\right| u_{n}+k_{2} v_{n} \text { in } \Omega_{T}, \\
\partial_{t} v_{n}-d \Delta v_{n}=-k_{2} v_{n}+k_{1} u_{n}+f \text { in } \Omega_{T} \\
\nabla u_{n} \cdot \nu=\nabla v_{n} \cdot \nu=0 \text { on } \Sigma_{T} \\
u_{n}(0, \cdot)=u_{0}, v_{n}(0, \cdot)=v_{0} \text { in } \Omega
\end{array}\right.
$$

Since the sequence $\left(u_{n}, v_{n}\right)_{n \geq 1}$ is bounded in $W^{2}$ and $\left(L^{\infty}\left([0, T] ; L^{2}(\Omega)\right)\right)^{2}$, there exists by the Aubin-Lions lemma a subsequence (not relabeled) such 
that

$$
\left\{\begin{array}{l}
u_{n} \rightarrow u \text { in } L^{2}\left(\Omega_{T}\right), \quad u_{n} \rightarrow u \text { a.e. in }\left(\Omega_{T}\right) \\
\nabla u_{n} \rightarrow \boldsymbol{\xi} \text { in }\left(L^{2}\left(\Omega_{T}\right)\right)^{3} \\
\partial_{t} u_{n} \rightarrow \psi \text { in } L^{2}\left(0, T,\left(H^{1}(\Omega)\right)^{\prime}\right) .
\end{array}\right.
$$

To prove that $\nabla u=\boldsymbol{\xi}$, we take a test function $\varphi \in\left(\mathcal{D}\left(\Omega_{T}\right)\right)^{3}$, so that:

$$
\int_{0}^{T} \int_{\Omega} \nabla u_{i}^{n} \varphi d x d t=-\int_{0}^{T} \int_{\Omega} u_{i}^{n} \nabla \varphi d x d t
$$

Taking the limit when $n \rightarrow \infty$ of both sides of this equation, we obtain

$$
\int_{0}^{T} \int_{\Omega} \boldsymbol{\xi}_{i} \varphi d x d t=-\int_{0}^{T} \int_{\Omega} u_{i} \nabla \varphi d x d t=\int_{0}^{T} \int_{\Omega} \nabla u_{i} \varphi d x d t
$$

and we conclude by a density argument. To prove that $\partial_{t} u=\psi$, we use a similar computation for the derivative with respect to time, with test function $\varphi \in C_{c}^{1}\left(0, T, H^{1}(\Omega)\right)$. Thus we have

$$
\begin{array}{r}
u_{n} \rightarrow u \text { in } L^{2}\left(0, T, H^{1}(\Omega)\right), \partial u_{n} \rightarrow \partial u \text { in } L^{2}\left(0, T,\left(H^{1}(\Omega)\right)^{\prime}\right) \\
\left|u_{n}\right| u_{n} \rightarrow|u| u \text { in } L^{2}\left(0, T, L^{\frac{3}{2}}(\Omega)\right),
\end{array}
$$

where the last assertion is a straightforward consequence of the upper bound of sequence $\left|u_{n}\right| u_{n}$ in $L^{2}\left(0, T, L^{\frac{3}{2}}(\Omega)\right)$ and the a.e. convergence of the sequence $\left(u_{n}\right)_{n \geq 1}$ in $\Omega_{T}$. We obtain also a similar convergence for $v_{n}$ towards $v$ as in (5.3).

Finally, thanks to suitable choices of test functions it follows that the limit function $v$ is solution of the following problem:

$$
\left\{\begin{array}{l}
\partial_{t} v-d \Delta v=-k_{2} v+k_{1} u+f \text { in } \Omega_{T} \\
\nabla v \cdot \nu=0 \text { on } \Sigma_{T} \\
v(0, \cdot)=v_{0} \text { in } \Omega
\end{array}\right.
$$

Obviously we prove that $\nabla v_{n} \rightarrow \nabla v$ in $\left(L^{2}\left(\Omega_{T}\right)^{3}\right.$ when $n \rightarrow \infty$ and thereby $v_{n}$ strongly converges to $v$ in $L^{2}\left(0, T, H^{1}(\Omega)\right)$. Since $h$ is continous, $z_{n} \rightarrow z$ in $L^{2}\left(\Omega_{T}\right)$ and $\nabla v_{n} \rightarrow \nabla v$ in $\left(L^{2}\left(\Omega_{T}\right)^{3}\right.$ there exists a subsequnce (not relabeled) such that $\tilde{h}\left(z_{n_{k}}\right) \nabla v_{n_{k}} \rightarrow \tilde{h}(z) \nabla v$ a.e. in $\Omega_{T}$. As $\tilde{h}\left(z_{n_{k}}\right) \nabla v_{n_{k}}$ is bounded in $L^{2}\left(\Omega_{T}\right)$ we obtain by the dominated convergence theorem :

$$
\tilde{h}\left(z_{n_{k}}\right) \nabla v_{n_{k}} \rightarrow \tilde{h}(z) \nabla v \text { in } L^{2}\left(\Omega_{T}\right),
$$


and we can pass to the limit in the $(\mathrm{P}-\mathrm{S})_{n}$ system. Thus

$$
\left\{\begin{array}{l}
\partial_{t} u-a \Delta u=-\beta \operatorname{div}(\tilde{h}(z) \nabla v)-k_{1} u-q|u| u+k_{2} v \quad \text { in } \Omega_{T}, \\
\partial_{t} v-d \Delta v=-k_{2} v+k_{1} u+f \quad \text { in } \Omega_{T}, \\
\nabla u \cdot \nu=\nabla v \cdot \nu=0 \quad \text { on } \Sigma_{T} \cdot, \\
u(0, \cdot)=u_{0}, v(0, \cdot)=v_{0} \quad \text { in } \Omega .
\end{array}\right.
$$

and we get $u=\Pi(z)$.

By the uniqueness of the solution $(u, v)$ of (5.5), we deduce that all the sequence converges. We conclude that $\Pi$ is a continuous mapping.

We can now apply the Schauder fixed point theorem to prove the existence statement of the following

Proposition 5.2. Let $f$ be a nonnegative function in $L^{2}\left(0, T ; L^{2}(\Omega)\right)$. For each couple of nonnegative functions $\left(u_{0}, v_{0}\right) \in\left(L^{2}(\Omega)\right)^{2}$ there exists a nonnegative solution for the problem $\left(P_{h}\right)$, with $h=\tilde{h}$.

To prove the positivity of the solution, we proceed as in section 4: we multiply the first equation by $-u^{-}$and the second by $-v^{-}$, we integrate in space and we add the two equations. Thanks to the identity $\int_{\Omega} h(u) \nabla v \nabla u^{-}=0$, a straightforward calculation gives:

$$
\frac{d}{d t}\left(\left\|u^{-}\right\|_{L^{2}(\Omega)}^{2}+\left\|v^{-}\right\|_{L^{2}(\Omega)}^{2}\right) \leq C\left(\left\|u^{-}\right\|_{L^{2}(\Omega)}^{2}+\left\|v^{-}\right\|_{L^{2}(\Omega)}\right),
$$

with $C>0$. We finish the proof by applying the Gronwall lemma.

For the uniqueness of solution of $\left(P_{h}\right)$ problem we have:

Theorem 5.3. Let $f \in L^{\infty}\left(\Omega_{T}\right)$ be a nonnegative function. Consider $\left(u_{0}, v_{0}\right) \in\left(L^{\infty}(\Omega)\right)^{2}$ such that $0 \leq u_{0} \leq M$ and $0 \leq v_{0}(x) \leq v_{M}$ almost everywhere in $\Omega$, where $v_{M}$ is a positive constant. Then there exists a constant $\alpha \geq 0$ such that

$$
0 \leq u(t, x) \leq M e^{\alpha t} \quad 0 \leq v(t, x) \leq v_{M} e^{\alpha t},
$$

and the solution of $\left(P_{h}\right)$ problem is unique, when $h=\tilde{h}$.

Proof. Let $\tilde{u}=u-M e^{\alpha t}$ and $\tilde{v}=v-v_{M} e^{\alpha t}$ then we have

$$
\begin{aligned}
& \tilde{u}_{t}=a \Delta \tilde{u}-\beta \nabla(h(u) \nabla \tilde{v})-k_{1} \tilde{u}-q \tilde{u}^{2}+k_{2} \tilde{v} \\
& -\left(\alpha M+k_{1} M+2 q u M-k_{2} v_{M}\right) e^{\alpha t}-q M^{2} e^{2 \alpha t},
\end{aligned}
$$


and

$$
\tilde{v}_{t}=a \Delta \tilde{v}+k_{1} \tilde{u}+\left\{f+e^{\alpha t}\left(\left(-k_{2}-\alpha\right) v_{M}+k_{1} M\right)\right\} .
$$

We take $\alpha$ large enough such that:

$$
f+e^{\alpha t}\left(\left(-k_{2}-\alpha\right) v_{M}+k_{1} M\right) \leq 0,
$$

and

$$
\alpha M+k_{1} M-k_{2} v_{M} \geq 0,
$$

Multiplying the first equation by $\tilde{u}^{+}$and the second by $\tilde{v}^{+}$and then adding the two equations gives:

$$
\frac{1}{2}\left(\frac{d}{d t}\left\|\tilde{u}^{+}\right\|_{L^{2}(\Omega)}^{2}+\frac{d}{d t}\left\|\tilde{v}^{+}\right\|_{L^{2}(\Omega)}^{2}\right) \leq \beta \int_{\Omega} h(u) \nabla v \nabla \tilde{u}^{+}+C\left(\left\|\tilde{u}^{+}\right\|_{L^{2}(\Omega)}^{2}+\left\|\tilde{v}^{+}\right\|_{L^{2}(\Omega)}^{2}\right)
$$

Thanks to (4.1) $\beta \int_{\Omega} h(u) \nabla v \nabla \tilde{u}^{+}=0$ and we obtain (5.6) by using Gronwall lemma.

To prove uniqueness, suppose that there exists two solutions $\left(u_{1}, v_{1}\right)$ and $\left(u_{2}, v_{2}\right)$. Then $\bar{u}=u_{1}-u_{2}$ and $\bar{v}=v_{1}-v_{2}$ verify

$$
\begin{aligned}
& \bar{u}_{t}=a \Delta \bar{u}-\beta \nabla\left(h\left(u_{1}\right) \nabla v_{1}-\left(h\left(u_{2}\right) \nabla v_{2}\right)-k_{1} \bar{u}-q u_{1}^{2}+q u_{2}^{2}+k_{2} \bar{v}\right. \\
& \bar{v}_{t}=d \Delta \bar{v}-k_{2} \bar{v}+k_{1} \bar{u} \\
& \bar{u}_{0}=\bar{v}_{0}=0 \text { a.e in } \Omega .
\end{aligned}
$$

Multiplying the first equation by $\bar{u}$, the second by $\bar{v}$ and integrating over $\Omega$ lead to

$$
\begin{aligned}
\frac{1}{2} \frac{d}{d t}\|\bar{u}\|_{L^{2}(\Omega)}^{2}+a\|\nabla \bar{u}\|_{L^{2}(\Omega)}^{2} \leq \beta \int_{\Omega} \mid\left(h\left(u_{1}\right) \nabla v_{1}\right. & \left.-h\left(u_{2}\right) \nabla v_{2}\right) \nabla \bar{u} \mid d x \\
& +C\left(\|\bar{u}\|_{L^{2}(\Omega)}^{2}+\|\bar{v}\|_{L^{2}(\Omega)}^{2}\right)
\end{aligned}
$$

and

$$
\frac{1}{2} \frac{d}{d t}\|\bar{v}\|_{L^{2}(\Omega)}^{2}++d\|\nabla \bar{v}\|_{L^{2}(\Omega)}^{2}+k_{2}\|\bar{v}\|_{L^{2}(\Omega)}^{2}=k_{1} \int_{\Omega} \bar{u} \bar{v} d x .
$$

It follows that

$$
\begin{gathered}
\frac{1}{2} \frac{d}{d t}\|\bar{u}\|_{L^{2}(\Omega)}^{2}+a\|\nabla \bar{u}\|_{L^{2}(\Omega)}^{2} \leq \beta \int_{\Omega}\left(\left|h\left(u_{1}\right)-h\left(u_{2}\right)\right|\left|\nabla v_{1}\right|+h\left(u_{2}\right)|\nabla \bar{v}|\right)|\nabla \bar{u}| d x \\
+C\left(\|\bar{u}\|_{L^{2}(\Omega)}^{2}+\|\bar{v}\|_{L^{2}(\Omega)}^{2}\right)
\end{gathered}
$$


and

$$
\frac{1}{2} \frac{d}{d t}\|\bar{v}\|_{L^{2}(\Omega)}^{2}+d\|\nabla \bar{v}\|_{L^{2}(\Omega)}^{2}+k_{2}\|\bar{v}\|_{L^{2}(\Omega)}^{2} \leq C\left(\|\bar{u}\|_{L^{2}}^{2}+\|\bar{v}\|_{L^{2}(\Omega)}^{2}\right)
$$

Recalling (5.6) $\bar{u}$ and $\bar{v}$ are bounded in $\Omega_{T}$. Classical parabolic regularity results and (5.4) imply that $v \in L^{p}\left(0, T, W^{2, p}(\Omega)\right)$ for each $p \in(1, \infty)$. By Sobolev embedding, there is $p>3$ such that $\nabla v_{1} \in L^{2}\left(0, T ; L^{\infty}(\Omega)\right)$. Hence

$$
\begin{array}{r}
\int_{\Omega}\left|h\left(u_{1}\right)-h\left(u_{2}\right)\right|\left|\nabla v_{1}\right||\nabla \bar{u}| d x \leq M\left\|\nabla v_{1}\right\|_{L^{\infty}(\Omega)}\|\bar{u}\|_{L^{2}(\Omega)}\|\nabla \bar{u}\|_{L^{2}(\Omega)} \\
\leq \varepsilon\|\nabla \bar{u}\|_{L^{2}(\Omega)}^{2}+C_{\varepsilon}\left\|\nabla v_{1}\right\|_{L^{\infty}(\Omega)}^{2}\|\bar{u}\|_{L^{2}(\Omega)}^{2}
\end{array}
$$

and

$$
\begin{aligned}
\int_{\Omega} h\left(u_{2}\right)|(\nabla \bar{v})||\nabla \bar{u}| & \leq \frac{M}{2}\|\nabla \bar{v}\|_{L^{2}(\Omega)}\|\nabla \bar{u}\|_{L^{2}(\Omega)} \\
& \leq \varepsilon^{\prime}\|\nabla \bar{u}\|_{L^{2}(\Omega)}^{2}+C_{\varepsilon}^{\prime}\|\nabla \bar{v}\|_{L^{2}(\Omega)}^{2}
\end{aligned}
$$

We sum up (5.9) and (5.8) multiplied by a constant $\sigma>0$ small enough, and we use (5.10) and (5.11) with a wise choise of $\varepsilon, \varepsilon^{\prime}$ and $\sigma$ such that: $\varepsilon+\varepsilon^{\prime} \leq a$ and $\sigma C_{\varepsilon}^{\prime} \leq d$. Thereby we prove the existence of a constant $C>0$ such that:

$$
\frac{d}{d t}\left(\sigma\|\bar{u}\|_{L^{2}(\Omega)}^{2}+\|\bar{v}\|_{L^{2}(\Omega)}^{2}\right) \leq C\left(\left\|\nabla v_{1}\right\|_{L^{\infty}(\Omega)}+1\right)\left(\sigma\|\bar{u}\|_{L^{2}(\Omega)}^{2}+\|\bar{v}\|_{L^{2}(\Omega)}^{2}\right)
$$

The Gronwall lemma entail that $\|\bar{u}(t)\|_{L^{2}(\Omega)}=\|\bar{v}(t)\|_{L^{2}(\Omega)}=0$ for every $t \in[0, T]$, which completes the proof.

\section{Acknowledgements.}

We thank DR Martial Bernoux - UMR Eco\&Sols (INRA, SupAgro, CIRAD, IRD) for his expertise and advice on soil carbon dynamics.

This research was financially supported by the Laboratories of Excellence (LabEx) NUMEV (solutions Numériques, Matricielles et Modélisation pour l'Environnement et le Vivant) and the LabEx CEMEB (Centre Méditerranéen de l'Environnement et de la Biodiversité). Acknowledgements are also extended to the Ecoles Doctorales SIBAGHE and I2S of Montpellier. 


\section{Appendix 1.}

\section{Non-emergence of spatial patterns in $\left(P_{h}\right)$ model without chemo- taxis term $(\beta=0)$.}

Firstly we considere the PDEs system $\left(P_{h}\right)$ without chemotaxis term $(\beta=$ $0)$. As in Lotka-Volterra systems [21], also known as the predator-prey equations, diffusion alone cannot disturb a constant equilibrium, and so spatial heterogeneity cannot emerge. Using the following notation:

$$
\tilde{x}=\sqrt{\frac{k_{1}}{a}} x \quad \tilde{t}=k_{1} t \quad \alpha=\frac{q}{k_{1}} \quad \zeta=\frac{k_{2}}{k_{1}} \quad c=\frac{f}{k_{1}} \quad D=\frac{d}{a}
$$

we obtain the following non-dimensional equations (we revoke the notation):

$$
\left\{\begin{array}{l}
\partial_{t} u=\Delta u-u-\alpha u^{2}+\zeta v \\
\partial_{t} v=D \Delta v+u-\zeta v+c
\end{array} \quad(x, t) \in \Omega \times(0 ; T)\right.
$$

with the same initial conditions and boundary conditions as $\left(P_{h}\right)$ system . Without diffusion, the system (1) has a unique positive steady state:

$$
u^{*}=\sqrt{\frac{c}{\alpha}} \quad v^{*}=\frac{u^{*}+c}{\zeta}
$$

To assess the steady state stability, the system is linearised around $\left(u^{*}, v^{*}\right)$. Setting:

$$
\varepsilon w_{1}=u-u^{*} \quad \varepsilon w_{2}=v-v^{*}
$$

where $0<\varepsilon \ll 1$, gives the following linear system:

$$
\left\{\begin{array}{l}
\partial_{t} w_{1}=\Delta w_{1}-w_{1}-2 \alpha u^{*} w_{1}+\zeta w_{2} \\
\partial_{t} w_{2}=D \Delta w_{2}+w_{1}-\zeta w_{2}
\end{array} \quad(x, t) \in \Omega \times(0, T),\right.
$$

with no-flux boundary conditions.

As in Murray [21], we looked for a solution of the form:

$$
\mathbf{w}=\left(\begin{array}{c}
w_{1} \\
w_{2}
\end{array}\right) \propto e^{(i \mathbf{k} \cdot \mathbf{x}+\rho t)} .
$$

Lets $k=|\mathbf{k}|$ be the Euclidean norm of the wave vector. We obtain the following eigenvalue problem:

$$
\mathbf{A w}=\rho \mathbf{w},
$$


where $\mathbf{A}$ is the two by two matrix

$$
\mathbf{A}=\left(\begin{array}{cc}
-1-2 \alpha u^{*}-k^{2} & \zeta \\
1 & -\zeta-k^{2} D
\end{array}\right)
$$

The eigenvalue $\rho$ depends on $k$.

Turing instability occurs (which means that spatial patterns appear) when $\rho\left(k^{2}\right)>0$, for a given value of $k$. But the matrix $\mathbf{A}$ has a strictly negative trace and a positive determinant, and so $\rho\left(k^{2}\right)<0$ for all values of $k$. Hence no patterns will emerge in this case.

\section{Emergence of spatial patterns in $\left(P_{h}\right)$ model with $\beta>0$.}

Finally, for the model with both diffusion and chemotaxis, it can be proven that the equilibrium solutions of the equation system $\left(P_{h}\right)$ can be rendered non-stable under certain conditions, and thus produce patterns and spatial heterogeneity. As in the previous section, the system $\left(P_{h}\right)$ was linearised around the steady state $\left(u^{*}, v^{*}\right)$. We obtain the following system

$$
\left\{\begin{array}{l}
\partial_{t} w_{1}=\Delta w_{1}-e \Delta w_{2}-w_{1}-2 \alpha u^{*} w_{1}+\zeta w_{2} \\
\partial_{t} w_{2}=D \Delta w_{2}+w_{1}-\zeta w_{2}
\end{array} \quad(x, t) \in \Omega \times(0, T),\right.
$$

where

$$
e=\beta h\left(u^{*}\right) \frac{k_{1}}{a} .
$$

Looking for solutions like in (4), the following eigenvalue problem must be solved:

$$
\mathbf{B} \mathbf{w}=\rho \mathbf{w},
$$

where $\mathbf{B}$ is the two by two matrix

$$
\mathbf{B}=\left(\begin{array}{cc}
-1-2 \alpha u^{*}-k^{2} & \zeta+e k^{2} \\
1 & -\zeta-k^{2} D
\end{array}\right)
$$

In this case, the trace of matrix $\mathbf{B}$ is strictly negative while its determinant can be strictly negative for some values of $k$. Thus, taking chemotaxis into account in the system may lead to the emergence of spatial patterns. 


\section{Appendix 2.}

\section{Numerical simulations.}

A set of validated parameters derived from studies published [7] was used to run numerical simulations. The data used came from an Andean Pramo site near Gavidia, Venezuela. As pattern geometries depend on the shape of the spatial domain [21], two different forms of spatial domain were tested. Figures below show the numerical simulations of the soil microbial biomass compartment for the nearly rectangular and circular domains, using either $h(u)=h_{1}(u)=u$ which does not prevent explicitly any overcrowding (Fig 1 and 2$)$, or $h(u)=h_{2}(u)=u(M-u)$ which explicitly does prevent overcrowding (Fig 3 and 4 ). These figures show the spatial variability and patterns obtained for soil microbial biomass after 60 days and for the two spatial domain shapes. The soil microbial biomass pattern agrees with the distribution within the soil matrix of the microbial hot spots at micron scale. Numerical simulations were performed using COMSOL Multiphysics 5.0.

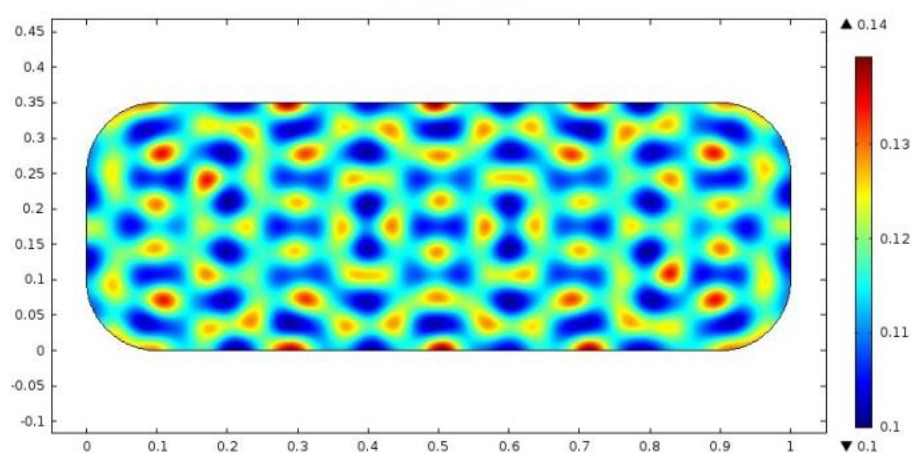

Figure 1: Spatial microbial biomass distribution when $h=h_{1}$ after 60 days. 


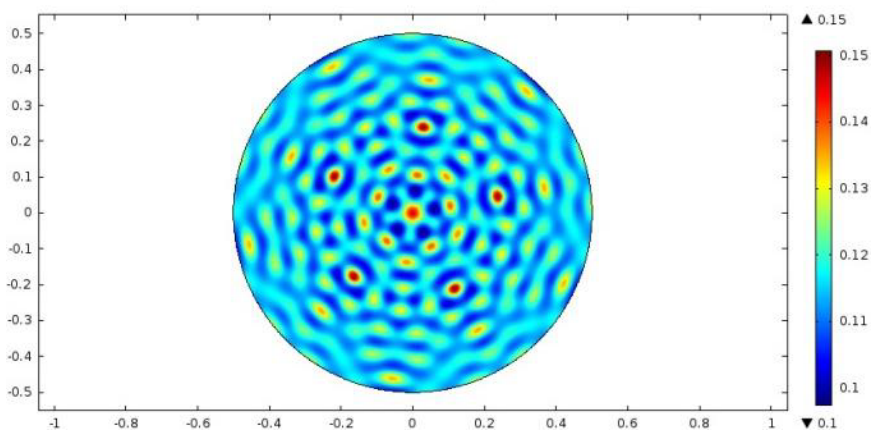

Figure 2: Spatial microbial biomass distribution when $h=h_{1}$ after 60 days.

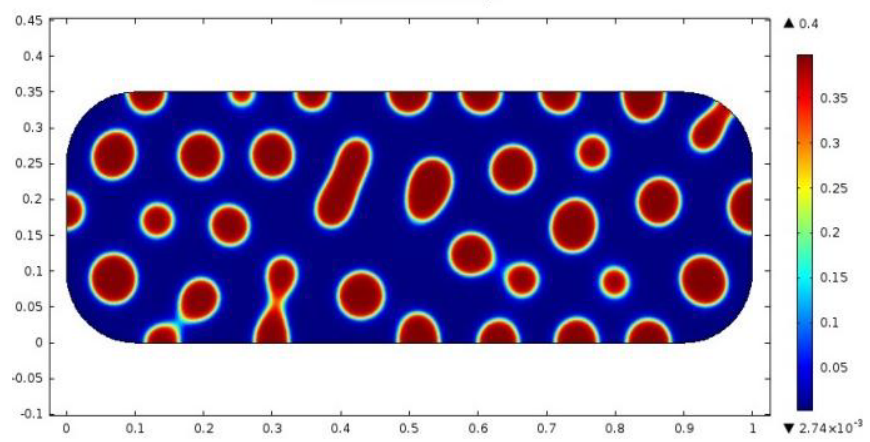

Figure 3: Spatial microbial biomass distribution when $h=h_{2}$ after 60 days.

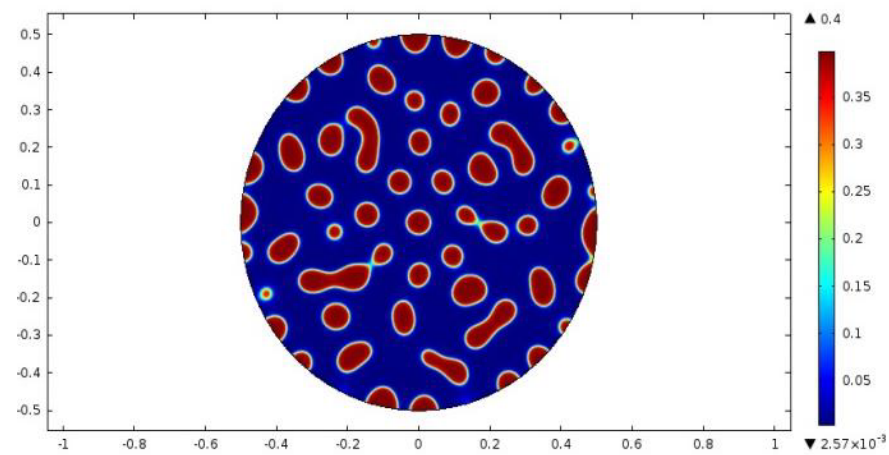

Figure 4: Spatial microbial biomass distribution when $h=h_{2}$ after 60 days. 


\section{References}

[1] Vogel C., Mueller C.W., Hschen C., Buegger F., Heister K., Schulz S., Schloter M., Kgel-Knabner I.: Submicron structures provide preferential spots for carbon and nitrogen sequestration in soils, Nature Communications, (2014), 5, no.2947.

[2] Manzoni, S., Porporato, A.: Soil carbon and nitrogen mineralization: Theory and models across scales. Soil Biology and Biochemistry, 41 (2009), p.1355-1379.

[3] Elzein A., Balesdent J.: Mechanistic Simulation of Vertical Distribution of Carbon Concentrations and Residence Times in Soils, Soil Science Society of America Journal, 59 (1995), p.1328-1335.

[4] Goudjo C., Leye B., Sy M.: Weak solution to a parabolic nonlinear system arising in biological dynamic in the soil, International Journal of Differential Equations, (2011).

[5] Deckmyn G. ,Campioli M., Muys B., Kraigher H.: Simulating C cycles in forest soils: Including the active role of micro-organisms in the ANAFORE forest model, Ecological Modelling, (2011), 222, p.19721985 .

[6] Pansu, M., Bottner, P., Sarmiento, L., Metselaar, K.: Comparison of five soil organic matter decomposition models using data from a $14 \mathrm{C}$ and 15N labeling field experiment. Global Biogeochemical Cycles, 18 (2004).

[7] Pansu, M., Sarmiento L., Rujano M.A., Ablan M., Acevedo D., Bottner P.: Modeling organic transformations by microorganisms of soils in six contrasting ecosystems: validation of the MOMOS model. Global Biogeochemical Cycles, 24 (2010).

[8] Hammoudi, A., Iosifescu, O., Bernoux, M.: Mathematical analysis of a nolinear model of soil carbon dynamics., Differ. Equ. Dyn. Syst., (2015) no 4, p. 453466.

[9] Hammoudi, A., Iosifescu, O., Bernoux, M.: Mathematical analysis of a spatially distributed soil carbon dynamics model., Analysis and Applications (2016), doi: 10.1142/SO219530516500081 
[10] Keller E.F., Segel L.A.: Initiation of slime mold aggregation viewed as an instability, J. Theor. Biol., (1970), 26, p.399 - 415.

[11] Bendahmane M.: Mathematical analysis of reaction-diffusion system modeling predator-prey with prey-taxis, Networks and Heterogeneous Media, (2008), 3(4), p.863-879.

[12] Ryu S.-U. , Yagi A. Optimal Control of KellerSegel Equations , Journal of Mathematical Analysis and Applications , (2001), 256(1), p.45-66.

[13] Osaki, K., Tsujikawa T., Yagi A., Mimura M.: Exponential Attractor for a Chemotaxis-growth System of Equations, Nonlinear Anal., (2002), 51(1), p.119-144.

[14] Hillen T., Painter K.: Global Existence for a Parabolic Chemotaxis Model with Prevention of Overcrowding, Advances in Applied Mathematics (2001),26(4), p.280-301.

[15] Wrzosek D.: Volume Filling Effect in Modelling Chemotaxis, Mathematical Modelling of Natural Phenomena, (2010), 5(1), p.123-147.

[16] Lions J. L.: Quelques Methodes de Resolution des Problemes aux Limites Non Lineaires. Dunod, 1969.

[17] Yagi A.: Abstract Parabolic Evolution Equations and their Applications, Springer-Verlag Berlin Heidelberg, 2010.

[18] Biler P., Hebisch W., Nadzieja T.: The Debye system: Existence and large time behavior of solutions, Nonlinear Anal., (1994), 238, p.1189 1209 .

[19] Temam R.: Navier-Stokes Equations. Theory and Numerical Analysis North-Holland, 1977.

[20] Eden A., Foias C., Nicolaenko B., Temam R.: Exponential attractors for dissipative evolution equations, Research in Applied Mathematics, Wiley New York, 1994.

[21] Murray J.D.: Mathematical Biology I: An Introduction, New-York: Springer , 2002

[22] Turing A.M.: The Chemical basis of Morphogenesis, Philosophical Transactions of the Royal Society of London, (1952), 237(641), p.3772 . 